\title{
On Vaccination Strategies for a SISV Epidemic Model Guaranteeing the Nonexistence of Endemic Solutions
}

\author{
S. Alonso-Quesada (iD, M. De la Sen $(\mathbb{D}$, and R. Nistal \\ Department of Electricity and Electronics, University of the Basque Country, UPV/EHU, Campus of Leioa, 48940 Leioa, Bizkaia, Spain \\ Correspondence should be addressed to M. De la Sen; manuel.delasen@ehu.eus
}

Received 12 June 2018; Accepted 23 August 2018; Published 2 October 2018

Academic Editor: Yong Zhou

Copyright (c) 2018 S. Alonso-Quesada et al. This is an open access article distributed under the Creative Commons Attribution License, which permits unrestricted use, distribution, and reproduction in any medium, provided the original work is properly cited.

\begin{abstract}
A vaccination strategy based on the state feedback control theory is proposed. The objective is to fight against the propagation of an infectious disease within a host population. This propagation is modelled by means of a SISV (susceptible-infectious-susceptiblevaccinated) epidemic model with a time-varying whole population and with a mortality directly associated with the disease. The vaccination strategy adds four free-design parameters, with three of them being the feedback gains of the vaccination control law. The other one is used to switch off the vaccination if the proportion of susceptible individuals is smaller than a prescribed threshold. The paper analyses the positivity of such a model under the proposed vaccination strategy as well as the conditions for the existence of the different equilibrium points of its normalized model. The fact that an appropriate adjustment of the control gains avoids the existence of endemic equilibrium points in the normalized SISV model while guaranteeing the existence of a unique disease-free equilibrium point being globally exponentially stable is proved. This is a relevant novelty dealt with in this paper. The persistence of the infectious disease within a host population irrespective of the growing properties of the whole population can be avoided in this way. Such theoretical results are mathematically proved and, also, they are illustrated by means of simulation examples. Moreover, the performance of the proposed vaccination strategy in several real situations is studied in some simulation examples. One of them deals with the presence of uncertainties, which affects the synthesis of the vaccination control law, in the measures of the subpopulations of the model.
\end{abstract}

\section{Introduction}

The use of mathematical models to describe the propagation of epidemic diseases has been broadly carried out for several decades $[1,2]$. The dynamics of such models can be used to take a decision about the convenience of applying control strategies, based on vaccination campaigns, quarantines, effective isolation measures in hospitals, and/or use of antiviral drugs among others in order to avoid the persistence of the disease or at least to minimize its effects within the host population [3-10]. The vaccination strategies can be governed by a signal synthesized using some control techniques such as state variables feedback, adaptive control, sliding mode control, or optimal control among others [3, 11-14]. Moreover, the control signal can be constant, timevarying function, impulsive, and so on [15-18].
A great variety of models have been used to study the propagation of infectious diseases. An important kind of such models is referred to as the class of compartmental models [19]. Such models split the total population in different categories depending on the status of the individuals with respect to the infection. The simplest one is the SIS epidemic model [20]. Such a model splits the population in two categories: susceptible (S) and infectious (I) subpopulations. There are two transitions in this model, one from the susceptible to the infectious category, which occurs with certain probability when contacts between susceptible and infectious individuals happen, and the other one from the infectious to the susceptible category once the infectious individual has passed an infectious period. Such a period as well as the probability of contagion of a susceptible individual after contacting with an infectious one is particular for each infectious disease. 
This kind of models assumes that the infectious individuals lose immunity and become susceptible immediately after recovering as it happens for sexually transmitted infections like chlamydia and gonorrhoea [21]. Also, the SIS model is appropriate for bacterial diseases such as meningitis and cholera. Moreover, there can be a mortality associated with each disease so that some of the infectious individuals may not recover from the disease. In this sense, the total population of the model can be considered as a constant if the mortality caused by the infectious disease and/or the disease time duration are small enough so that such an assumption be acceptable. Otherwise, a time-varying population has to be considered to properly study the dynamics of the infectious disease $[17,22]$. There are other compartmental models, such as SIR and SEIR ones, which are widely used in the literature $[11,12,14-16,18,19,22-24]$. The SIR one adds a subpopulation of recovered $(\mathrm{R})$ by immunization individuals. Such a category includes individuals who have recovered from the disease after passing the infectious period and the immunization can be for life or for a certain limited period. Once such an immunization period has ended, the recovered individuals come back to the susceptible category. This model is properly referred to as SIRS model [25]. The SEIR model adds a new subpopulation with respect to the SIR one, namely, the exposed (E) one. Such a category includes the susceptible individuals who have been infected after contacts with infectious individuals but they do not present symptoms and they do not transmit the disease after contacting with susceptible individuals. A vaccination (V) subpopulation can be added to all of the aforementioned epidemic models. Such models are known, respectively, as SISV, SVIR, and SVEIR models [26-30]. The vaccination subpopulation includes the individuals who have received a vaccine to prevent the contagion of the disease. Other models consider the application of treatment by drugs to a fraction of the infective individuals. Then, the infective subpopulation is split into two categories, namely, the infective without treatment (I) and the infective under treatment $(\mathrm{T})$. The individuals within the first one can transmit the disease after contacts, so that they are infectious, whereas the individuals in the other one cannot propagate the disease [31]. All the aforementioned models only consider the direct transmission of the disease after direct contacts between the susceptible and infectious individuals. However, there are also models describing the propagation of vector-transmitted diseases as well as models for indirectly transmitted diseases via pathogens shed in the environment by the infectious individuals $[32,33]$.

In this paper, the analysis of a SISV epidemic model for directly transmitted diseases, with a time-varying population and mortality directly associated with the disease, under a vaccination strategy based on a state feedback control law is carried out. The model assumes that the susceptible individuals who receive a vaccine pass directly to the vaccinated subpopulation and they maintain the immunity for life although they have contacts with infectious individuals. Also, an efficiency of $100 \%$ of the vaccines is assumed, which implies that all of the susceptible individuals who receive a vaccine pass to the vaccinated category. The vaccination strategy provides some free-design parameters, namely, the three constant control gains, with each one associated with each state variable of the model, and an additional one which switches off the vaccination when the proportion of susceptible individuals is smaller than a prescribed threshold. A similar SISV model is analysed in $[26,30]$ under a vaccination rate being proportional to the number of susceptible individuals. The main differences and novelties of the current paper with respect to them are focused on the synthesis of the vaccination control law, namely, the following:

(a) The control law is based on the feedback of all the variables of the model so that there are three control constant gains to be tuned instead of just one used in $[26,30]$.

(b) Some of the control gains can take negative values but if the feedback control signal becomes negative at some time instant, then such a signal is zeroed. Precisely, an appropriate choice for the values of such gains is key to guarantee the inexistence of endemic equilibrium (EE) points and in this way to achieve the eradication of the infection. This is an important novelty of this paper.

(c) Another difference with respect to [26] is that the current paper deals with a time-varying whole population and a mortality directly induced by the disease.

(d) A different with respect to [30], where the whole population is also time-varying with a mortality related to the infection, is that in the current paper the growing properties of the total population and the infection are separated by the control design. In this sense the infection can be asymptotically removed even if the total population is unbounded as time increases due to the higher rate of births related to the natural death rate. Note that this is the current situation for the whole entire world population. However, the model proposed in [30] under vaccination converges to a disease-free equilibrium (DFE) point or an EE one where the total population is a constant value.

The analysis of the current paper includes the proof of the positivity of the model under the proposed vaccination strategy. Also the influence of the control gains on the dynamics of the disease transmission within the host population is studied by means of a normalized SISV model. Such a normalized model has two independent state variables, instead of the three of the original SISV model, which simplifies the analysis. The conditions for the existence of the equilibrium points of this normalized model depending on the assigned values to the control gains are analysed. In this context, the existence of appropriate choices for the control gains guaranteeing the nonexistence of EE points jointly with the existence of a unique globally exponentially stable DFE point for the normalized SISV model is proved. Such a fact is a key result to achieve the main objective of the paper, namely, the eradication of the disease propagation within the host population, while guaranteeing the persistence of the whole population, by means of the application of a vaccination strategy based on the proposed state feedback control law with an appropriate adjustment of the control gains. Furthermore, 
the proportion of the vaccinated subpopulation in the aforementioned globally exponentially stable DFE point depends on the values of the control gains. Then, the number of vaccines to be used during the vaccination campaign can be prefixed by adjusting such gains. In a practical situation, this fact can be used to choose the control gains according to the number of available vaccines while guaranteeing the unique existence of the globally asymptotically stable DFE point.

Several simulation examples are carried out to complement the theoretical results of the paper. The first one shows that an infectious disease, with a mortality directly associated with the illness, can lead to the extinction of the host population in absence of some prevention action. This fact motivates the application of a vaccination campaign to avoid such an extinction. In this context, the second example shows that the application of a vaccination campaign based on the proposed state feedback control law, with an appropriate adjustment of the control gains, achieves the main objective of eradicating the infectious disease while guaranteeing the growing of the host population. Such an example illustrates the main theoretical results of the paper. The third example points out that some specifications of the vaccination campaign, such as the number of required vaccines and the duration of the campaign, depend on the values assigned to the control gains. Finally, the two last examples show that a vaccination campaign based on the proposed strategy could be implemented in a real situation. The fourth example takes into account that the number of vaccines to be injected at each day has to be an integer number. In this context, two alternative ways for designing the vaccination campaign with such a restriction are dealt with. On the other hand, the fifth example takes into account the fact that the measures of the state variables of the model can be subject to uncertainties since the knowledge of the exact number of susceptible, infectious, and vaccinated individuals at each time instant is not possible in a real situation [34, 35]. In this context, this example considers the lack of precision in the measures of the subpopulations affecting the synthesis of the control law. The result shows that the control law is robust under the presence of small uncertainties in the measures. In summary, the main contributions of the paper are as follows: (i) the design of a control law via the feedback of all the state variables of the SISV epidemic model where some of the control gains can take negative values while maintaining the control signal nonnegative for all time, (ii) the proof that an appropriate adjustment of the control gains guarantees the nonexistence of EE points and the existence of a unique globally exponentially stable DFE point in the normalized SISV model which leads to the eradication of the infectious disease, (iii) the study of the influence of the control gains values in the proportions of the subpopulations in the globally exponentially stable DFE point as well as in the transient behavior of the controlled model by means of a simulation example, and (iv) the presentation of a method to implement a vaccination campaign based on the proposed control technique in a realistic situation with uncertainties in the subpopulations measures needed to synthesize the control law and a simulation example to illustrate it.
The paper is organized as follows. Section 2 describes the SISV model and the vaccination strategy based on a state feedback control law. Section 3 deals with the analysis of the positivity of the SISV model under the proposed vaccination. Also, the analysis of the equilibrium points of its normalized model related to the control gains is carried out. Such a study points out that a vaccination strategy based on feedback of the model variables can achieve the eradication of the disease with an appropriate choice of the values for the control gains. Section 4 deals with numerical examples which illustrate the theoretical results and proposes several ways of implementing a vaccination campaign based on the presented strategy in a realistic situation. Finally, Section 5 ends the paper with some concluding remarks.

\section{The SISV Epidemic Model}

The SISV epidemic model splits the host population into three different categories: susceptible, infectious, and vaccinated subpopulations. The transitions between the subpopulation categories of this epidemic model are given by the following differential equations:

$$
\begin{aligned}
& \dot{S}(t)=v N(t)+\gamma I(t)-\beta \frac{S(t) I(t)}{N(t)}-\mu S(t)-U(t) \\
& \dot{I}(t)=\beta \frac{S(t) I(t)}{N(t)}-(\gamma+\alpha+\mu) I(t) \\
& \dot{V}(t)=-\mu V(t)+U(t)
\end{aligned}
$$

subject to $S(0) \geq 0, I(0) \geq 0, V(0) \geq 0$, and $S(0)+I(0)+$ $V(0)>0$, where $S(t), I(t)$, and $V(t)$ denote, respectively, the susceptible, infectious, and vaccinated subpopulations at the instant $t$ and $N(t)=S(t)+I(t)+V(t)$ denotes the whole population. By summing up the equations of (1) one obtains the dynamics of the whole population given by

$$
\dot{N}(t)=(v-\mu) N(t)-\alpha I(t) .
$$

The parameters of the model are all strictly positive. Namely, $v$ and $\mu$ denote, respectively, the birth and the mortality by natural causes rates of the host population. The transmission of the infection from mothers to sons/daughters is not considered so the new births are included directly in the susceptible category. The rest of the parameters are associated with the infectious disease: $\beta$ denotes the infection transmission rate, $\gamma$ is the recuperation rate, and $\alpha$ is the mortality by causes directly related to the disease. The inverse of $\gamma$ denotes the average time interval that an infectious individual spends within the infectious category before passing to the susceptible category. The transition of an individual from the susceptible category to the infectious one can happen, with certain probability, when such a susceptible individual contacts an infectious one. In this sense, the factor $\beta I(t) / N(t)$ is the per capita probability of acquiring the infection at the 
instant $t$ and the term $\beta S(t) I(t) / N(t)$ represents the total rate of transmissions of the infection at the instant $t$ [1].

The function $U(t)$ denotes a control signal based on the application of a vaccination strategy. It is assumed that the application of a vaccine to a susceptible individual transfers such an individual to the vaccinated category and he/she/it is maintained in such a status until he/she/it dies by natural causes. Such a fact is taken into account by the inclusion of the term $-\mu V(t)$ in the dynamics of the vaccinated subpopulation. In other words, an efficiency of $100 \%$ in the vaccination and that the vaccinated individuals acquire immunization for life are assumed.

\section{Remark 1 .}

(i) The dynamics of the whole population depends on the net growth rate $r=v-\mu$ in the absence of disease. It implies that the whole population exponentially increases if $v>\mu$, it holds constant to $N(0) \forall t$ if $v=\mu$, or it exponentially decreases to zero and the host population is extinguished if $v<\mu$. This form of population dynamics is called exponential birth and deaths. An alternative way of modelling the dynamics of the whole population consists in replacing the term $v N(t)$ by a constant recruitment rate $A$. Such a form is referred to as constant immigration with exponential death rate [20].

(ii) The propagation of the infectious disease is described by a SIS epidemic model composed by the two first equations of (1) in the absence of vaccination, i.e., if $U(t)=0 \forall t \geq 0$, and with an initial condition without vaccinated individuals, i.e., if $V(0)=0$. Direct calculations show that such a model has two possible equilibrium points depending on the value of the basic reproduction number $R_{0}=\beta /(v+\gamma+\alpha)$ [16]. If $R_{0} \leq 1$, the model has a unique equilibrium point at which all the population is susceptible. Such an equilibrium point is referred to as DFE point since there are not infectious individuals. Furthermore, such a point is globally asymptotically stable so that the disease is eradicated from the host population irrespective of the initial condition. Otherwise, if $R_{0}>$ 1 , the model has two equilibrium points: the DFE point, which is unstable, and an EE point at which there is a proportion of the whole population in the susceptible status, $s_{e e}^{*}=(v+\gamma) /(\beta-\alpha)$, and the other one in the infectious category, $\iota_{e e}^{*}=(\beta-$ $(v+\gamma+\alpha)) /(\beta-\alpha)$. Furthermore, such an EE point is globally asymptotically stable so that the disease persists within the host population irrespective of the initial condition. Such properties are proved in [16] for the case of whole population dynamics with constant immigration and exponential death rate. However, such properties can be also proved for a whole population dynamics with exponential birth and deaths, as proposed in the current paper, by using a normalized version of the SIS model built from applying the variable changes $s(t)=S(t) / N(t)$ and $\iota(t)=I(t) / N(t)$ to such a model.

\section{Design of Vaccination Strategies Based on Control Theory}

The application of vaccination strategies can be considered in two situations: (i) when the propagation of the infectious disease leads to an EE point or (ii) when the propagation dynamics converges to a DFE point but an improvement in the transient behavior is required. The first situation is going to be dealt with in this section. In this sense, an infectious disease whose propagation is described by the epidemic model (1), where its parameters satisfy the condition $\beta>$ $v+\gamma+\alpha$, is considered. This means that the basic reproduction number of the model without considering the vaccination subpopulation (SIS model) fulfills $R_{0}>1$. The vaccination is applied to a fraction of the susceptible individuals. Such individuals pass to the category referred to as vaccinated subpopulation when they receive the vaccine.

A vaccination based on a control signal defined by

$$
\begin{aligned}
& U(t) \\
& = \begin{cases}\max \left\{0, k_{s} S(t)+k_{i} I(t)+k_{v} V(t)\right\} & \text { if } S(t) \geq \varepsilon_{s} N(t)>0 \\
0 & \text { otherwise }\end{cases}
\end{aligned}
$$

is considered where $k_{s}, k_{i}$, and $k_{v}$ are real constants, namely, the controller gains. Such a law is based on the feedback of the variables of the SISV epidemic model while it is nonnegative definite. Furthermore, the vaccination is suspended while the proportion of susceptible subpopulation is strictly smaller than a prescribed strictly positive threshold $\varepsilon_{s} \in(0,1)$.

Theorem 2 (positivity of the SISV model). The SISV epidemic model (1) is positive under the application of the control signal (3) irrespective of the values assigned to the control gains since $S(t) \geq 0, I(t) \geq 0$, and $V(t) \geq 0 \forall t \geq 0$ provided that $S(0) \geq 0$, $I(0) \geq 0$, and $V(0) \geq 0$.

Proof. Assume that there exists a finite time instant $t_{1}>0$ such that $S(t) \geq 0$ and $V(t) \geq 0 \forall t \in\left[0, t_{1}\right]$ with $I\left(t_{1}\right)<0$. The continuity of the SISV model together with $S(0) \geq 0, I(0) \geq 0$ and $V(0) \geq 0$ implies that there exists some time instant $t_{I}<$ $t_{1}$ such that $I\left(t_{I}\right)=0$ with $S\left(t_{I}\right) \geq 0$ and $V\left(t_{I}\right) \geq 0$. It implies that $\dot{I}\left(t_{I}\right)=0$ and then $I(t)=I\left(t_{I}\right)=0 \forall t \geq t_{I}$ from (1). Then, the starting assumption $I\left(t_{1}\right)<0$ at a finite time instant $t_{1}>0$ is not true. Now, assume that there exists a finite time instant $t_{2}>0$ such that $S(t) \geq 0$ and $I(t) \geq 0 \forall t \in\left[0, t_{2}\right]$ with $V\left(t_{2}\right)<0$. The continuity of the SISV model together with $S(0) \geq 0, I(0) \geq 0$, and $V(0) \geq 0$ implies that there exists some time instant $t_{V}<t_{2}$ such that $V\left(t_{V}\right)=0$ with $S\left(t_{V}\right) \geq 0$ and $I\left(t_{V}\right) \geq 0$. This implies that $\dot{V}\left(t_{V}\right)=U\left(t_{V}\right) \geq 0$ from (1) and (3). Then, the starting assumption $V\left(t_{2}\right)<0$ at a finite time instant $t_{2}>0$ is not true. Finally, assume that there exists a finite time instant $t_{3}>0$ such that $I(t) \geq 0$ and $V(t) \geq 0 \forall t \in\left[0, t_{3}\right]$ with $S\left(t_{3}\right)<0$. The continuity of the SISV model together with the conditions $S(0) \geq 0, I(0) \geq 0$, and $V(0) \geq 0$ implies that there exists some time instant $t_{S}<$ $t_{3}$ such that $S\left(t_{S}\right)=0$ with $I\left(t_{S}\right) \geq 0$ and $V\left(t_{S}\right) \geq 0$. It follows that $\dot{S}\left(t_{S}\right)=(v+\gamma) I\left(t_{S}\right)+v V\left(t_{S}\right) \geq 0$ from (1) and (3). Then the starting assumption $S\left(t_{3}\right)<0$ at some finite time instant $t_{3}>0$ is not true. In summary, there are not time instants 
at which $S(t), I(t)$, or $V(t)$ take negative values provided that $S(0) \geq 0, I(0) \geq 0$, and $V(0) \geq 0$. The result of the theorem is proved.

3.1. Normalized SISV Model. A variable change lets us obtain a normalized SISV epidemic model useful to analyse the dynamics of the propagation of the disease under the proposed vaccination strategy. Such a variable change is given by

$$
\begin{aligned}
& s(t)=\frac{S(t)}{N(t)} \\
& \iota(t)=\frac{I(t)}{N(t)} \\
& v(t)=\frac{V(t)}{N(t)}
\end{aligned}
$$

where the resulting new variables $s(t), \iota(t)$, and $v(t)$ represent, respectively, the proportion of susceptible, infectious, and vaccinated individuals within the host population. Note that $s(t) \in[0,1], \iota(t) \in[0,1]$, and $v(t) \in[0,1] \forall t \geq 0$ are derived from Theorem 2 if a vaccination strategy based on the control law (3) is applied in the epidemic model.

One obtains the following normalized SISV model:

$$
\begin{aligned}
\dot{s}(t) & =v-v s(t)+\gamma \iota(t)-(\beta-\alpha) s(t) \iota(t)-u(t) \\
i(t) & =-(v+\gamma+\alpha) \iota(t)+\alpha \iota^{2}(t)+\beta s(t) \iota(t) \\
\dot{v}(t) & =-v v(t)+\alpha \iota(t) v(t)+u(t)
\end{aligned}
$$

by applying the aforementioned variable change in (1) where

$$
\begin{aligned}
& u(t)=\frac{U(t)}{N(t)} \\
& = \begin{cases}\max \left\{0, k_{s} s(t)+k_{i} \iota(t)+k_{v} v(t)\right\} & \text { if } s(t) \geq \varepsilon_{s}>0 \\
0 & \text { otherwise }\end{cases}
\end{aligned}
$$

denotes the normalized control signal. Model (5) under the control law (6) can be written as

$$
\begin{aligned}
i(t)= & (\beta-(v+\gamma+\alpha)) \iota(t)-(\beta-\alpha) \iota^{2}(t) \\
& -\beta \iota(t) v(t) \\
\dot{v}(t)= & -v v(t)+\alpha \iota(t) v(t)+u(t)
\end{aligned}
$$

by taking into account the fact that $s(t)+\iota(t)+v(t)=1 \forall t \geq 0$. The following subsection analyses the equilibrium points for model (5) under the control law (6) depending on the values of the controller gains.

3.1.1. Equilibrium Points. The normalized SISV model (5) under the control law (6) asymptotically reaches an equilibrium point given by $\iota^{*}, v^{*}$, and $s^{*}=1-\iota^{*}-v^{*}$ when $i(t)=0$ and $\dot{v}(t)=0$. Then

$$
\begin{aligned}
(\beta-(v+\gamma+\alpha)) \iota^{*}-(\beta-\alpha) \iota^{* 2}-\beta \iota^{*} v^{*} & =0 \\
-v v^{*}+\alpha \iota^{*} v^{*}+u^{*} & =0
\end{aligned}
$$

where $u^{*}$ denotes the value of the control signal at some potential equilibrium point. Such a value can be $u^{*}=k_{s}+$ $\left(k_{i}-k_{s}\right) \iota^{*}+\left(k_{v}-k_{s}\right) v^{*}>0$ or $u^{*}=0$ by taking into account the control law (6).

Assume that $u^{*}=0$. Then, the second equation of (8) has two solutions, namely, either $v^{*}=0$ or $\iota^{*}=v / \alpha$. The first equation of (8) has two solutions when $v^{*}=0$, namely, $\iota^{*}=0$ and $\iota^{*}=(\beta-(v+\gamma+\alpha)) /(\beta-\alpha)$. Both of them satisfy that $\iota^{*} \in[0,1]$ from the fact that $\beta>v+\gamma+\alpha>\alpha$ so that they are compatible with the positivity of the SISV model established in Theorem 2 . Note that such a property implies that a feasible equilibrium point of the normalized SISV model has to have $\iota^{*} \in[0,1]$. On the other hand, the first equation of (8) has a unique solution in $v^{*}$ when $\iota^{*}=v / \alpha$, namely, $v^{*}=1-(\beta v+\alpha(\gamma+\alpha)) / \beta \alpha$. From the positivity of the SISV model, such a solution is only valid if the model parameters fulfil the conditions $v \leq \alpha$ and $\beta \geq \alpha(\alpha+\gamma) /(\alpha-v)$ so that $\iota^{*} \in[0,1]$ and $v^{*} \in[0,1]$, respectively, as the positivity of the SISV model requires. In summary, the normalized SISV model has potentially three equilibrium points assuming that $u^{*}=0$, one of them is a DFE point (DFE1) and the others are EE points (EE1 and EE2); namely,

$$
\text { DFE } 1: \iota^{*}=0 ; v^{*}=0 ; s^{*}=1
$$

$E E 1: \quad \iota^{*}=\frac{\beta-(v+\gamma+\alpha)}{\beta-\alpha} ; \quad v^{*}=0 ; \quad s^{*}=\frac{v+\gamma}{\beta-\alpha}$

EE2: $\iota^{*}=\frac{v}{\alpha} ; \quad v^{*}=1-\frac{\beta v+\alpha(\gamma+\alpha)}{\beta \alpha} ; s^{*}=\frac{\alpha+\gamma}{\beta}$.

The feasibility of such points requires that $u^{*}=0$ at them. In this context, the point DFE1 exists if the control gain $k_{s}$ is chosen such that $k_{s} \leq 0$ so that $u^{*}=0$ at it. Moreover, such an existence is irrespective of the values chosen for the control gains $k_{i}$ and $k_{v}$. Such facts are deduced from (6) by taking into account the fact that $s^{*}=1>\varepsilon_{s}$. On the other hand, the existence of the point EE1 requires that $s^{*}=(v+$ $\gamma) /(\beta-\alpha)<\varepsilon_{s}$ or, otherwise, that the controller gains $k_{s}$ and $k_{i}$ are chosen such that $k_{i}(\beta-(v+\gamma+\alpha))+k_{s}(v+\gamma) \leq 0$ so that $k_{s}+\left(k_{i}-k_{s}\right) \iota^{*}+\left(k_{v}-k_{s}\right) v^{*} \leq 0$ and then $u^{*}=0$ at such a point by taking into account (6). Finally, the existence of the point EE2 requires that $s^{*}=(\alpha+\gamma) / \beta<\varepsilon_{s}$ or, otherwise, that the controller gains $k_{s}, k_{i}$, and $k_{v}$ are chosen such that $k_{s} \alpha(\alpha+\gamma)+k_{i} \beta v+k_{v}(\beta(\alpha-v)-\alpha(\alpha+\gamma)) \leq 0$ so that $k_{s}+$ $\left(k_{i}-k_{s}\right) \iota^{*}+\left(k_{v}-k_{s}\right) v^{*} \leq 0$ and then $u^{*}=0$ at such a point by taking into account (6).

Now, assume that $u^{*}=k_{s}+\left(k_{i}-k_{s}\right) \iota^{*}+\left(k_{v}-k_{s}\right) v^{*} \geq 0$. The equation system (8) has three potential solutions. One of them with $\iota^{*}=0$ and the others with $\iota^{*}=\iota_{j} \neq 0$ where $\iota_{j}, j \in$ $\{1,2\}$, denotes the potential solutions of $\iota_{j}^{2}+g_{1}\left(k_{s}, k_{i}, k_{v}\right) \iota_{j}+$ 
$g_{0}\left(k_{s}, k_{v}\right)=0$ with the coefficients $g_{0}\left(k_{s}, k_{v}\right)$ and $g_{1}\left(k_{s}, k_{i}, k_{v}\right)$, depending on the controller gains, defined as follows.

$$
\begin{aligned}
& g_{0}\left(k_{s}, k_{v}\right) \\
& \quad=\frac{\left(v-k_{v}\right)(\beta-(v+\gamma+\alpha))-k_{s}(v+\gamma+\alpha)}{\alpha(\beta-\alpha)} \\
& g_{1}\left(k_{s}, k_{i}, k_{v}\right) \\
& \quad=\frac{\beta\left(k_{v}-k_{i}-v-\alpha\right)+\alpha\left(2 v+\gamma+\alpha+k_{s}-k_{v}\right)}{\alpha(\beta-\alpha)}
\end{aligned}
$$

The substitution of $\iota^{*}=0$ in the second equation of (8) implies that $v^{*}=k_{s} /\left(v+k_{s}-k_{v}\right)$ so that the model has a potential DFE point, namely, the point DFE2 given by if $k_{v}-k_{s} \neq v$. Otherwise, i.e., if $k_{v}-k_{s}=v$, such an equilibrium point does not exist except in the particular case that simultaneously $k_{s}=0$ and $k_{v}=v$.

In the general case that $k_{v}-k_{s} \neq v$, the feasibility of the point DFE2 requires that $v^{*} \in[0,1], s^{*} \in[0,1]$, and $u^{*} \geq$ 0 . Such conditions are simultaneously satisfied if the control gains $k_{s}$ and $k_{v}$ are chosen so that $\varepsilon_{s} \leq\left(v-k_{v}\right) /\left(k_{s}-k_{v}+v\right) \leq$ 1 . This implies that the point DFE2 exists if (i) $k_{s}>0$ and $k_{v} \leq v-\varepsilon_{s} k_{s} /\left(1-\varepsilon_{s}\right)$, (ii) $k_{s}<0$ and $k_{v} \geq v-\varepsilon_{s} k_{s} /\left(1-\varepsilon_{s}\right)$, or (iii) $k_{s}=0$ while $k_{v} \neq v$. Note that the point DFE2 is the same as DFE1 in the last case.

On the other hand, each one of the solutions of $\iota_{j}^{2}+$ $g_{1}\left(k_{s}, k_{i}, k_{v}\right) \iota_{j}+g_{0}\left(k_{s}, k_{v}\right)=0$, if they exist, is feasible equilibrium point for the model if $i_{j} \in[0,1]$. In such a case, the model has one or two EE points given by

$$
\text { DFE2: } \iota^{*}=0 ; v^{*}=\frac{k_{s}}{k_{s}-k_{v}+v} ; s^{*}=\frac{v-k_{v}}{k_{s}-k_{v}+v}
$$

$$
\begin{aligned}
& \text { EE3: } \quad \iota^{*}=\iota_{1} ; \quad v^{*}=v_{1}=\frac{(\beta-\alpha)\left(1-\iota_{1}\right)-(v+\gamma)}{\beta} ; s^{*}=s_{1}=1-\iota_{1}-v_{1}=\frac{\alpha\left(1-\iota_{1}\right)+v+\gamma}{\beta} \\
& \text { EE4: } \iota^{*}=\iota_{2} ; \quad v^{*}=v_{2}=\frac{(\beta-\alpha)\left(1-\iota_{2}\right)-(v+\gamma)}{\beta} ; s^{*}=s_{2}=1-\iota_{2}-v_{2}=\frac{\alpha\left(1-\iota_{2}\right)+v+\gamma}{\beta} .
\end{aligned}
$$

The feasibility of the point EE3 and, respectively, EE4, requires that $\iota_{j} \in[0,1], v_{j} \in[0,1]$, and $s_{j} \in[0,1]$ for $j=1$ and, respectively, $j=2$, while the value of the control signal at such a point fulfils $u^{*}=u_{1} \geq 0$ and, respectively, $u^{*}=u_{2} \geq 0$. The four conditions for the feasibility of the point EE3 and, respectively, EE4, are jointly fulfilled if $\iota_{j} \in\left[0, \min \left\{(\beta-(v+\gamma+\alpha)) /(\beta-\alpha),\left(v+\gamma+\alpha-\varepsilon_{s} \beta\right) / \alpha\right\}\right]$ and the controller gains $k_{s}, k_{i}$, and $k_{v}$ are chosen such that $\left(\beta\left(k_{v}-k_{i}\right)-\alpha\left(k_{v}-k_{s}\right)\right) \iota_{j} \leq k_{s}(v+\gamma+\alpha)+k_{v}(\beta-(v+\gamma+\alpha))$ for $j=1$ and, respectively, $j=2$, as it can be deduced by direct calculations. Note that there are not solutions for $\iota_{j}$ satisfying simultaneously the four conditions $\iota_{j} \in[0,1], v_{j} \in[0,1]$, $s_{j} \in[0,1]$, and $u^{*}=u_{j} \geq 0$ if the free-design control parameter $\varepsilon_{s}$ is chosen such that $\varepsilon_{s}>(v+\gamma+\alpha) / \beta$. Then, the points EE3 and EE4 do not exist in such a situation. However, the normalized model has at least one EE point, namely, the point EE2, under such a choice for the parameter $\varepsilon_{s}$.

In the particular case that $k_{s}=0$ and $k_{v}=v$ simultaneously, the system of equations (8) becomes as follows.

$$
\begin{aligned}
(\beta-(v+\gamma+\alpha)) \iota^{*}-(\beta-\alpha) \iota^{* 2}-\beta \iota^{*} v^{*} & =0 \\
\alpha \iota^{*} v^{*}+k_{i} \iota^{*} & =0
\end{aligned}
$$

The second equation of (13) has two solutions, namely, $\iota^{*}=0$ for any $v^{*}$ and $v^{*}=-k_{i} / \alpha$ for any $\iota^{*} \neq 0$. By introducing such solutions in the first equation of (13), one obtains the following equilibrium points for the model.

$$
\begin{gathered}
\text { DFE3: } \iota^{*}=0 ; \quad v^{*} \in[0,1] ; s^{*} \in[0,1] \text { such that } s^{*}+v^{*}=1 \\
\text { EE5: } \iota^{*}=\frac{\alpha(\beta-(v+\gamma+\alpha))+\beta k_{i}}{\alpha(\beta-\alpha)} ; v^{*}=-\frac{k_{i}}{\alpha} ; s^{*}=\frac{v+\gamma-k_{i}}{\beta-\alpha}
\end{gathered}
$$

The feasibility of the equilibrium points defined by DFE3 requires that the values of the control signal at such points fulfil $u^{*} \geq 0$. Such a condition is fulfilled if $s^{*} \geq \varepsilon_{s}$ by taking into account the fact that $u^{*}=k_{v} v^{*}=v v^{*}>0$ from the control law (6). Then the set of feasible points DFE3 is as follows. 
Note that the point DFE1 is included in the set of points DFE3. On the other hand, the feasibility of the point EE5 requires that $\iota^{*} \in[0,1], v^{*} \in[0,1]$, and $s^{*} \in[0,1]$ as well as that the value of the control signal at such a point fulfils $u^{*} \geq 0$. Such conditions are jointly fulfilled if the control gain $k_{i}$ is chosen such that $\max \{-\alpha(\beta-(v+\gamma+\alpha)) / \beta,(\beta(v-\alpha)+\alpha(\gamma+\alpha)) / \beta\} \leq$ $k_{i} \leq \min \left\{0, v+\gamma-\varepsilon_{s}(\beta-\alpha)\right\}$ while $k_{i} \neq 0$. The existence of a nonzero range for the values of $k_{i}$ so that the point EE5 is feasible requires that the model parameters satisfy $\beta(v-\alpha)+$ $\alpha(\gamma+\alpha) \leq 0$ while the parameter $\varepsilon_{s}$ of the control law satisfies $\varepsilon_{s} \leq(v+\gamma) /(\beta-\alpha)$. Moreover, the point EE5 is the same as EE1 if $k_{i}=0$ and its feasibility is guaranteed irrespective if either $\varepsilon_{s} \leq(v+\gamma) /(\beta-\alpha)$ or $\varepsilon_{s}>(v+\gamma) /(\beta-\alpha)$ since $u^{*}=0$ at the point EE1 (or EE5) while $k_{s}=0, k_{i}=0$, and $k_{V}=v$.

Table 1 summarizes the equilibrium points of the model and their feasibility conditions for the different assignments of the controller gains. The following theorem establishes the conditions that the control parameters have to satisfy for guaranteeing the inexistence of EE points in the normalized SISV model. In this way, the infectious disease can be eradicated from the host population.

Theorem 3 (conditions for inexistence of EE points in the normalized SISV model). The normalized SISV model (5) under the control law (6) does not have EE points if the parameter $\varepsilon_{s}$ of the control law (3), or equivalently (6), satisfies the condition

(c1) $0<\varepsilon_{s} \leq \min \{(\alpha+\gamma) / \beta,(v+\gamma) /(\beta-\alpha)\}$

and the control gains $k_{s}, k_{i}$, and $k_{v}$ simultaneously satisfy

(c2) $k_{i}(\beta-(v+\gamma+\alpha))+k_{s}(v+\gamma)>0$,

(c3) $k_{s}<0$ or $k_{i} \neq 0$,

(c4) if $\beta(v-\alpha)+\alpha(\alpha+\gamma) \leq 0$ then $k_{s} \neq 0$ or $k_{v} \neq v$,

(c5) $k_{s} \alpha(\alpha+\gamma)+k_{i} \beta v+k_{v}(\beta(\alpha-v)-\alpha(\alpha+\gamma))>0$,

(c6) at least one of the following conditions:

(i) $k_{i} \geq m_{i 1}\left(k_{v}\right)$ and $m_{s 1}\left(k_{v}\right) \leq k_{s} \leq m_{s 2}\left(k_{i}, k_{v}\right)$,

(ii) $k_{i} \leq m_{i 2}\left(k_{v}\right)$ and $m_{s 3}\left(k_{i}, k_{v}\right) \leq k_{s} \leq m_{s 1}\left(k_{v}\right)$,

(iii) $k_{s}>\max \left\{m_{s 4}\left(k_{i}, k_{v}\right), m_{s 3}\left(k_{i}, k_{v}\right)\right\}$,

(iv) $k_{s}<\min \left\{m_{s 4}\left(k_{i}, k_{v}\right), m_{s 2}\left(k_{i}, k_{v}\right)\right\}$,

where

$$
\begin{aligned}
m_{i 1}\left(k_{v}\right)= & \frac{v+\gamma}{v+\gamma+\alpha} k_{v}+\alpha-\frac{v(v+\gamma)}{v+\gamma+\alpha} \\
& +\frac{\alpha\left(v^{2}-\alpha^{2}+\gamma(2 v+\gamma)\right)}{\beta(v+\gamma+\alpha)} \\
m_{i 2}\left(k_{v}\right)= & \frac{v+\gamma}{v+\gamma+\alpha} k_{v}-\alpha-\frac{v(v+\gamma)}{v+\gamma+\alpha} \\
& +\frac{\alpha\left((\gamma+\alpha)^{2}+v(v+2 \gamma+2 \alpha)\right)}{\beta(v+\gamma+\alpha)}
\end{aligned}
$$

$$
\begin{aligned}
m_{s 1}\left(k_{v}\right)= & \frac{\beta-(v+\gamma+\alpha)}{v+\gamma+\alpha}\left(v-k_{v}\right) ; \\
m_{s 2}\left(k_{i}, k_{v}\right)= & \frac{\beta}{\alpha} k_{i}-\frac{\beta-\alpha}{\alpha} k_{v} \\
& +\frac{\beta(v-\alpha)+\alpha(\alpha-2 v-\gamma)}{\alpha} \\
m_{s 3}\left(k_{i}, k_{v}\right)= & \frac{\beta}{\alpha} k_{i}-\frac{\beta-\alpha}{\alpha} k_{v} \\
& +\frac{\beta(v+\alpha)-\alpha(2 v+\gamma+\alpha)}{\alpha} ; \\
m_{s 4}\left(k_{i}, k_{v}\right)= & k_{v}-\frac{\beta}{v+\gamma} k_{i}+\alpha-v .
\end{aligned}
$$

As a consequence, only the points DFE1, DFE2, and DFE3 are feasible under such conditions.

Proof. The results summarized in the feasibility conditions column of Table 1 are used for this proof. In this way, the points EE1 and EE5 do not exist under the conditions (c1), (c2), (c3), and (c4), where (c4) is only necessary if the model parameters are such that $\beta(v-\alpha)+\alpha(\alpha+\gamma) \leq 0$. Moreover, the conditions (c1) and (c5) avoid the existence of the point EE2. Finally, the points EE3 and EE4 do not exist if at least one of the conditions of (c6) is satisfied. Concretely, any of such conditions imply that $\iota_{j} \notin[0,1]$ as it is proved in the following way. The proportions of infectious subpopulation at the potential equilibrium points EE3 and EE4 are the solutions of the equation $\iota_{j}^{2}+g_{1}\left(k_{s}, k_{i}, k_{v}\right) \iota_{j}+g_{0}\left(k_{s}, k_{v}\right)=0$ with $g_{0}\left(k_{s}, k_{v}\right)$ and $g_{1}\left(k_{s}, k_{i}, k_{v}\right)$ defined in (10). Note that the function $F\left(\iota_{j}\right)=\iota_{j}^{2}+g_{1}\left(k_{s}, k_{i}, k_{v}\right) \iota_{j}+g_{0}\left(k_{s}, k_{v}\right)$, for any given values of $k_{s}, k_{i}$, and $k_{v}$, corresponds to a parabola which is opening to the top and its intersecting points with the abscissas axis are the solutions of $\iota_{j}^{2}+g_{1}\left(k_{s}, k_{i}, k_{v}\right) \iota_{j}+$ $g_{0}\left(k_{s}, k_{v}\right)=0$. In this context, direct calculations prove that such intersecting points are not within the domain $[0,1]$ under any of the conditions (c6). First, note that

$$
\begin{aligned}
& F(0)=g_{0}\left(k_{s}, k_{v}\right) ; \\
& F(1)=1+g_{1}\left(k_{s}, k_{v}, k_{i}\right)+g_{0}\left(k_{s}, k_{v}\right) ; \\
& \iota_{j, \text { min }}=-\frac{1}{2} g_{1}\left(k_{s}, k_{v}, k_{i}\right)
\end{aligned}
$$

where $\iota_{j, \text { min }}$ is the value of $\iota_{j}$ at which the parabola $F\left(\iota_{j}\right)$ reaches its minimum value. Under the condition (i) of (c6) one obtains that

$$
\begin{aligned}
& F(0)=\frac{\left(v-k_{v}\right)(\beta-(v+\gamma+\alpha))-k_{s}(v+\gamma+\alpha)}{\alpha(\beta-\alpha)} \\
& \leq \frac{\left(v-k_{v}\right)(\beta-(v+\gamma+\alpha))-m_{s 1}(v+\gamma+\alpha)}{\alpha(\beta-\alpha)}=0
\end{aligned}
$$


TABLE 1: Equilibrium points and their feasibility conditions.

\begin{tabular}{|c|c|c|}
\hline $\begin{array}{l}\text { Equilibrium } \\
\text { point }\end{array}$ & Proportion of subpopulations & Feasibility conditions \\
\hline DFE1 & $s^{*}=1 ; \iota^{*}=0 ; v^{*}=0$ & $k_{s} \leq 0$, any $k_{i}$ and $k_{v}$ \\
\hline DFE2 & $\begin{array}{l}s^{*}=\frac{v-k_{v}}{k_{s}-k_{v}+v} ; \iota^{*}=0 ; v^{*}= \\
\frac{k_{s}}{k_{s}-k_{v}+v}\end{array}$ & $\begin{array}{l}\text { any } k_{i} \text { with } k_{s} \text { and } k_{v} \text { such that } \\
\text { (i) } k_{s}>0 \text { and } k_{v} \leq v-\varepsilon_{s} k_{s} /\left(1-\varepsilon_{s}\right) \text { or } \\
\text { (ii) } k_{s}<0 \text { and } k_{v} \geq v-\varepsilon_{s} k_{s} /\left(1-\varepsilon_{s}\right)\end{array}$ \\
\hline DFE3 & $\begin{array}{l}s^{*} \in\left[\varepsilon_{s}, 1\right] ; \iota^{*}=0 ; v^{*} \in\left[0,1-\varepsilon_{s}\right] \\
\text { such that } s^{*}+v^{*}=1\end{array}$ & $k_{s}=0$, any $k_{i}$ and $k_{v}=v$ \\
\hline EE1 & $s^{*}=\frac{v+\gamma}{\beta-\alpha} ; \iota^{*}=\frac{\beta-(v+\gamma+\alpha)}{\beta-\alpha} ; v^{*}=0$ & $\begin{array}{l}\text { (i) any } k_{s}, k_{i} \text { and } k_{v} \text { if } \varepsilon_{s}>(v+\gamma) /(\beta-\alpha) \text { or } \\
\text { (ii) any } k_{v} \text { with } \\
\text { (1) } k_{s} \geq 0 \text { and } k_{i}=0 \text { or } \\
\text { (2) } k_{s} \text { and } k_{i} \text { fulfilling } k_{i}(\beta-(v+\gamma+\alpha))+k_{s}(v+\gamma) \leq 0 \\
\text { if } \varepsilon_{s} \leq(v+\gamma) /(\beta-\alpha)\end{array}$ \\
\hline EE2 & $\begin{array}{l}s^{*}=\frac{\alpha+\gamma}{\beta} ; \iota^{*}=\frac{v}{\alpha} ; v^{*}= \\
1-\frac{\beta v+\alpha(\gamma+\alpha)}{\beta \alpha}\end{array}$ & $\begin{array}{l}\text { (i) any } k_{s}, k_{i} \text { and } k_{v} \text { if } \varepsilon_{s}>(\alpha+\gamma) / \beta \text { or } \\
\text { (ii) } k_{s}, k_{i} \text { and } k_{v} \text { such that } \\
k_{s} \alpha(\alpha+\gamma)+k_{i} \beta v+k_{v}(\beta(\alpha-v)-\alpha(\alpha+\gamma)) \leq 0 \\
\text { otherwise }\end{array}$ \\
\hline $\begin{array}{l}\text { EE3 } \\
\text { and/or } \\
\text { EE4 }\end{array}$ & $\begin{array}{l}s^{*}=s_{j}=\frac{\alpha\left(1-\iota_{j}\right)+v+\gamma}{\beta} ; \iota^{*}=\iota_{j} \\
v^{*}=v_{j}=\frac{(\beta-\alpha)\left(1-\iota_{j}\right)-(v+\gamma)}{\beta} \\
\iota_{j} \text { solution of } \iota_{j}^{2}+g_{1}\left(k_{s}, k_{i}, k_{v}\right) \iota_{j}+ \\
g_{0}\left(k_{s}, k_{v}\right)=0\end{array}$ & $\begin{array}{l}\iota_{j} \in\left[0, \min \left\{(\beta-(v+\gamma+\alpha)) /(\beta-\alpha),\left(v+\gamma+\alpha-\varepsilon_{s} \beta\right) / \alpha\right\}\right] \text { with } \\
k_{s}, k_{i} \text { and } k_{v} \text { such that } \\
\left(\beta\left(k_{v}-k_{i}\right)-\alpha\left(k_{v}-k_{s}\right)\right) \iota_{j} \leq k_{s}(v+\gamma+\alpha)+k_{v}(\beta-(v+\gamma+\alpha))\end{array}$ \\
\hline EE5 & $\begin{array}{l}s^{*}=\frac{v+\gamma-k_{i}}{\beta-\alpha} ; \iota^{*}= \\
\frac{\alpha(\beta-(v+\gamma+\alpha))+\beta k_{i}}{\alpha(\beta-\alpha)} ; v^{*}=-\frac{k_{i}}{\alpha}\end{array}$ & $\begin{array}{l}k_{s}=0, k_{v}=v \text { with } k_{i} \text { such that } \\
k_{i} \leq \min \left\{0, v+\gamma-\varepsilon_{s}(\beta-\alpha)\right\} \text { and simultaneously } \\
k_{i} \geq \max \left\{-\frac{\alpha(\beta-(v+\gamma+\alpha))}{\beta}, \frac{\beta(v-\alpha)+\alpha(\gamma+\alpha)}{\beta}\right\} \\
\text { which is possible if } \\
\beta(v-\alpha)+\alpha(\gamma+\alpha) \leq 0 \text { and } \varepsilon_{s} \leq(v+\gamma) /(\beta-\alpha)\end{array}$ \\
\hline
\end{tabular}

$$
\begin{aligned}
& \iota_{j, \min }=-\frac{\beta\left(k_{v}-k_{i}-v-\alpha\right)+\alpha\left(2 v+\gamma+\alpha+k_{s}-k_{v}\right)}{2 \alpha(\beta-\alpha)} \\
& \geq \frac{\beta k_{i}-(\beta-\alpha) k_{v}+\beta(v+\alpha)-\alpha(2 v+\gamma+\alpha)-\alpha m_{s 2}}{2 \alpha(\beta-\alpha)} \\
& =\frac{\beta(v+\alpha)-\alpha(2 v+\gamma+\alpha)-\beta(v-\alpha)-\alpha(\alpha-2 v-\gamma)}{2 \alpha(\beta-\alpha)} \\
& =1
\end{aligned}
$$

where the condition $k_{i} \geq m_{i 1}\left(k_{v}\right)$ is necessary to guarantee $m_{s 1}\left(k_{v}\right) \leq m_{s 2}\left(k_{i}, k_{v}\right)$ and then the existence of values for $k_{s}$ such that $m_{s 1}\left(k_{v}\right) \leq k_{s} \leq m_{s 2}\left(k_{i}, k_{v}\right)$ is possible, as one can deduce by direct calculations. The result $\iota_{j \text {,min }} \geq 1$ implies that $F\left(\iota_{j}\right)$ is monotonically decreasing $\forall \iota_{j} \in[0,1)$. Such a fact, together with $F(0) \leq 0$, implies that $F\left(\iota_{j}\right)<0 \forall \iota_{j} \in(0,1]$ since the parabola $F\left(\iota_{j}\right)$ is opening to the top. Then, $F\left(\iota_{j}\right)=0$ cannot have solutions within $\iota_{j} \in[0,1]$ except $\iota_{j}=0$ under such a condition (i) of (c6) and that solution corresponds to a DFE point.

Under the condition (ii) of (c6) one obtains that

$$
F(0)=\frac{\left(v-k_{v}\right)(\beta-(v+\gamma+\alpha))-k_{s}(v+\gamma+\alpha)}{\alpha(\beta-\alpha)}
$$

$$
\begin{aligned}
& \geq \frac{\left(v-k_{v}\right)(\beta-(v+\gamma+\alpha))-m_{s 1}(v+\gamma+\alpha)}{\alpha(\beta-\alpha)}=0 \\
& \iota_{j, \text { min }}=-\frac{\beta\left(k_{v}-k_{i}-v-\alpha\right)+\alpha\left(2 v+\gamma+\alpha+k_{s}-k_{v}\right)}{2 \alpha(\beta-\alpha)} \\
& \leq \frac{\beta k_{i}-(\beta-\alpha) k_{v}+\beta(v+\alpha)-\alpha(2 v+\gamma+\alpha)-\alpha m_{s 3}}{2 \alpha(\beta-\alpha)} \\
& =0
\end{aligned}
$$

where the condition $k_{i} \leq m_{i 2}\left(k_{v}\right)$ is necessary to guarantee $m_{s 3}\left(k_{i}, k_{v}\right) \leq m_{s 1}\left(k_{v}\right)$ and then the existence of values for $k_{s}$ such that $m_{s 3}\left(k_{i}, k_{v}\right) \leq k_{s} \leq m_{s 1}\left(k_{v}\right)$ is possible, as one can deduce by direct calculations. The result $\iota_{j \text {,min }} \leq 0$ implies that $F\left(\iota_{j}\right)$ is monotonically increasing $\forall \iota_{j} \in(0,1]$. Such a fact, together with $F(0) \geq 0$, implies that $F\left(\iota_{j}\right)>0 \forall \iota_{j} \in(0,1]$ since the parabola $F\left(\iota_{j}\right)$ is opening to the top. Then, $F\left(\iota_{j}\right)=0$ cannot have solutions within $\iota_{j} \in[0,1]$ except $\iota_{j}=0$ under such a condition (ii) of (c6) and that solution corresponds to a DFE point. 
Under the condition (iii) of (c6) one obtains that

$$
\begin{aligned}
F(1) & =1+\frac{-(v+\gamma) k_{s}-\beta k_{i}+(v+\gamma) k_{v}-\beta \alpha+\alpha(2 v+\gamma+\alpha)-v(v+\gamma+\alpha)}{\alpha(\beta-\alpha)} \\
& =\frac{-(v+\gamma) k_{s}-\beta k_{i}+(v+\gamma) k_{v}+(\alpha-v)(v+\gamma)}{\alpha(\beta-\alpha)}<\frac{-(v+\gamma) m_{s 4}-\beta k_{i}+(v+\gamma) k_{v}+(\alpha-v)(v+\gamma)}{\alpha(\beta-\alpha)}=0 \\
\iota_{j, \text { min }} & =-\frac{\beta\left(k_{v}-k_{i}-v-\alpha\right)+\alpha\left(2 v+\gamma+\alpha+k_{s}-k_{v}\right)}{2 \alpha(\beta-\alpha)}<\frac{\beta k_{i}-(\beta-\alpha) k_{v}+\beta(v+\alpha)-\alpha(2 v+\gamma+\alpha)-\alpha m_{s 3}}{2 \alpha(\beta-\alpha)}=0 .
\end{aligned}
$$

The result $\iota_{j \text {,min }}<0$ implies that $F\left(\iota_{j}\right)$ is monotonically increasing $\forall \iota_{j} \in[0,1]$. Such a fact, together with $F(1)<0$, implies that $F\left(\iota_{j}\right)<0 \forall \iota_{j} \in[0,1]$ since the parabola $F\left(\iota_{j}\right)$ is opening to the top. As a consequence, there are not solutions for $F\left(\iota_{j}\right)=0$ with $\iota_{j} \in[0,1]$.

Finally, under the condition (iv) of (c6) one obtains that

$$
\begin{aligned}
& F(1)=\frac{-(v+\gamma) k_{s}-\beta k_{i}+(v+\gamma) k_{v}+(\alpha-v)(v+\gamma)}{\alpha(\beta-\alpha)} \\
& >\frac{-(v+\gamma) m_{s 4}-\beta k_{i}+(v+\gamma) k_{v}+(\alpha-v)(v+\gamma)}{\alpha(\beta-\alpha)} \\
& =0
\end{aligned}
$$$$
\iota_{j, \min }=-\frac{\beta\left(k_{v}-k_{i}-v-\alpha\right)+\alpha\left(2 v+\gamma+\alpha+k_{s}-k_{v}\right)}{2 \alpha(\beta-\alpha)}
$$$$
>\frac{\beta k_{i}-(\beta-\alpha) k_{v}+\beta(v+\alpha)-\alpha(2 v+\gamma+\alpha)-\alpha m_{s 2}}{2 \alpha(\beta-\alpha)}
$$

$$
=1 \text {. }
$$

The result $\iota_{j \text {,min }}>1$ implies that $F\left(\iota_{j}\right)$ is monotonically decreasing $\forall \iota_{j} \in[0,1]$. Such a fact, together with $F(1)>0$, implies that $F\left(\iota_{j}\right)>0 \forall \iota_{j} \in[0,1]$ since the parabola $F\left(\iota_{j}\right)$ is opening to the top. As a consequence, there are not solutions for $F\left(\iota_{j}\right)=0$ with $\iota_{j} \in[0,1]$.

In summary, there are not feasible solutions for EE points under the conditions established in the theorem and the result is proved.

The following theorem analyses the local stability of the DFE points of the normalized SISV model under the control law (6).

Theorem 4 (local stability/instability of the DFE points of the normalized SISV model).

(i) The point DFE1 is locally exponentially unstable whenever it exists, i.e., when $\beta>v+\gamma+\alpha$ and $k_{s} \leq 0$ is chosen in the control law (6). (ii) The point DFE2 is locally exponentially stable if the control gains $k_{s}$ and $k_{v}$ in (6) satisfy

$$
\begin{gathered}
k_{s}>0 ; \\
v-\frac{v+\gamma+\alpha}{\beta-(v+\gamma+\alpha)} k_{s}<k_{v} \leq v-\frac{\varepsilon_{s}}{1-\varepsilon_{s}} k_{s}
\end{gathered}
$$

while $\varepsilon_{s}<(v+\gamma+\alpha) / \beta$. On the other hand, the point DFE2 is locally exponentially unstable if $k_{s}$ and $k_{v}$ satisfy

$$
\begin{aligned}
& k_{s}<0 \\
& k_{v} \geq v-\frac{\varepsilon_{s}}{1-\varepsilon_{s}} k_{s} .
\end{aligned}
$$

(iii) The point DFE3 is locally unstable if $v^{*}<1-(v+\gamma+$ $\alpha) / \beta$. Otherwise, i.e., if $v^{*} \geq 1-(v+\gamma+\alpha) / \beta$, the point DFE3 is critically stable while the control parameter $\varepsilon_{s}$ in (6) is chosen such that $\varepsilon_{s} \leq(v+\gamma+\alpha) / \beta$.

Proof. (i) The feasibility of the point DFE1 requires that $k_{s} \leq$ 0 as it has been pointed out in Table 1. The Jacobi matrix associated with the linearized model around such a point is given by

$$
J_{D F E 1}=\left[\begin{array}{cc}
\beta-(v+\gamma+\alpha) & 0 \\
0 & -v
\end{array}\right]
$$

by taking into account the fact that $u(t)=0$ in the neighbourhood of such an equilibrium point from (6). One of the eigenvalues of such a matrix is a strictly positive real under the condition $\beta>v+\gamma+\alpha$ so the DFE1 point is locally exponentially unstable.

(ii) The feasibility of the point DFE2 requires that either $k_{s}>0$ and $k_{v} \leq v-\varepsilon_{s} k_{s} /\left(1-\varepsilon_{s}\right)$ or $k_{s}<0$ and $k_{v} \geq v-$ $\varepsilon_{s} k_{s} /\left(1-\varepsilon_{s}\right)$ as it has been pointed out in Table 1 . The Jacobi matrix associated with the linearized model around such a point is given by

$$
\begin{aligned}
& J_{D F E 2} \\
& =\left[\begin{array}{cc}
\beta-(v+\gamma+\alpha)-\beta \frac{k_{s}}{v-k_{v}+k_{s}} & 0 \\
\alpha \frac{k_{s}}{v-k_{v}+k_{s}}+k_{i}-k_{s} & -v+k_{v}-k_{s}
\end{array}\right]
\end{aligned}
$$


by taking into account the fact that $u(t)=k_{s}+\left(k_{i}-k_{s}\right) \iota(t)+$ $\left(k_{v}-k_{s}\right) v(t)>0$ in the neighbourhood of such an equilibrium point from (6). In the case that $k_{s}>0$ and $k_{v} \leq v-\varepsilon_{s} k_{s} /\left(1-\varepsilon_{s}\right)$ the eigenvalues $\lambda_{1}$ and $\lambda_{2}$ of $J_{D F E 2}$ satisfy that

$$
\begin{aligned}
\lambda_{1}= & \beta-(v+\gamma+\alpha)-\beta \frac{k_{s}}{v-k_{v}+k_{s}} \\
< & \beta-(v+\gamma+\alpha) \\
& -\beta \frac{k_{s}}{((v+\gamma+\alpha) /(\beta-(v+\gamma+\alpha))) k_{s}+k_{s}} \\
= & 0 \\
\lambda_{2}= & -v+k_{v}-k_{s} \leq-\frac{\varepsilon_{s} k_{s}}{1-\varepsilon_{s}}-k_{s}=-\frac{k_{s}}{1-\varepsilon_{s}}<0
\end{aligned}
$$

by taking into account conditions (23). Then, both eigenvalues of $J_{D F E 2}$ are strictly negative real so that the point DFE2 is locally exponentially stable. Note that the condition $\varepsilon_{s}<$ $(v+\gamma+\alpha) / \beta$ is necessary so that a nonempty domain of values for the gain $k_{v}$ fulfilling the condition (23) exists. On the other hand, in the case that $k_{s}<0$ and $k_{v} \geq v-\varepsilon_{s} k_{s} /\left(1-\varepsilon_{s}\right)$, the eigenvalue $\lambda_{2}$ of $J_{D F E 2}$ satisfies that

$$
\lambda_{2}=-v+k_{v}-k_{s} \geq-\frac{\varepsilon_{s} k_{s}}{1-\varepsilon_{s}}-k_{s}=-\frac{k_{s}}{1-\varepsilon_{s}}>0
$$

by taking into account conditions (24). Then, at least one of the eigenvalues of $J_{D F E 2}$ is strictly positive real so that the point DFE2 is locally exponentially unstable.

(iii) The feasibility of the point DFE3 requires that $k_{s}=0$ and $k_{v}=v$ as it has been pointed out in Table 1 . Moreover, the proportions of subpopulations at such an equilibrium point fulfil $s^{*} \in\left[\varepsilon_{s}, 1\right], \iota^{*}=0$ and $v^{*} \in\left[0,1-\varepsilon_{s}\right]$. Such conditions together with the positivity property of the SISV epidemic model imply that $u(t)=k_{s}+\left(k_{i}-k_{s}\right) \iota(t)+\left(k_{v}-k_{s}\right) v(t)=k_{i} \iota(t)+$ $v v(t)>0$ in the neighbourhood of such an equilibrium point from the control law (6). Then, the Jacobi matrix associated with the linearized model around such a point is given by

$$
J_{D F E 3}=\left[\begin{array}{cc}
\beta-(v+\gamma+\alpha)-\beta v^{*} & 0 \\
\alpha v^{*}+k_{i} & 0
\end{array}\right] .
$$

One of the eigenvalues is irrespective of the value of $v^{*}$, namely, $\lambda_{2}=0$. The other one, namely, $\lambda_{1}$, depends on the value of $v^{*}$. In this context, if $v^{*}<1-(v+\gamma+\alpha) / \beta$, then the point DFE3 is locally unstable since $\lambda_{1}>0$. Otherwise, i.e., if $v^{*} \geq 1-(v+\gamma+\alpha) / \beta$, the point DFE3 is locally critically stable since $\lambda_{1} \leq 0$. Note that the condition $\varepsilon_{s} \leq(v+\gamma+\alpha) / \beta$ is necessary so that a nonempty domain of values for $v^{*}$ fulfilling the condition $1-(v+\gamma+\alpha) / \beta \leq v^{*} \leq 1-\varepsilon_{s}$ exists.

Remark 5. The following results are derived from Theorems 2,3 , and 4 and they are of relevant interest in the vaccination design context:

(i) Assume that the free-design parameter $\varepsilon_{s}$ and the control gains $k_{s}, k_{i}$, and $k_{v}$ simultaneously satisfy the conditions of Theorem 3 so that the normalized SISV model does not have EE points. If, furthermore, (a) $k_{s}<0$ and $k_{v}<v-\varepsilon_{s} k_{s} / 1-\varepsilon_{s}$, then the model only has a DFE point, concretely, the DFE1 one. Moreover, such a point is locally exponentially unstable.

(b) $k_{s}<0$ and $k_{v} \geq v-\varepsilon_{s} k_{s} / 1-\varepsilon_{s}$, then the points DFE1 and DFE2 coexist. Moreover, both points are locally exponentially unstable.

(c) $k_{s}=0$ and $k_{v}=v$, then the points DFE1 and DFE3 coexist. The point DFE1 is locally exponentially unstable and the local stability of the point DFE3 depends on the proportion of vaccinated subpopulation at such an equilibrium point. In this sense, if $v^{*} \geq 1-$ $(v+\gamma+\alpha) / \beta$, the point DFE3 is locally critically stable; otherwise, it is locally unstable. One obtains that the dynamics of the normalized infectious subpopulation around the point DFE3 is given by $i=[\beta-(v+$ $\left.\gamma+\alpha)-\beta v^{*}\right] \iota$ in view of the Jacobi matrix $J_{D F E 3}$ in (29). Then, the time evolution of the infectious proportion is given by $\iota(t)=\iota\left(t_{0}\right) e^{\lambda_{1}\left(t-t_{0}\right)}$ around such an equilibrium point with $\lambda_{1}=\beta-(v+\gamma+\alpha)-\beta v^{*}$ and $t_{0}$ denoting the time instant at which the model state goes in the neighbourhood of the point DFE3. On the other hand, the dynamics of the normalized vaccinated subpopulation around the point DFE3 is given by $\dot{v}=\left(\alpha v^{*}+k_{i}\right) \iota$ in view of the Jacobi matrix $J_{D F E 3}$. Then, the time evolution of the vaccinated proportion is given by $v(t)=v\left(t_{0}\right)-\left(\alpha v^{*}+k_{i}\right)(1-$ $\left.e^{\lambda_{1}\left(t-t_{0}\right)}\right) \iota\left(t_{0}\right) / \lambda_{1}$ around such an equilibrium point. In this way, if $v^{*}>1-(v+\gamma+\alpha) / \beta$, then $\lambda_{1}<0$ so that the infectious proportion converges to zero while the vaccination proportion converges to the constant value $v_{\infty}=v\left(t_{0}\right)-\left(\alpha v^{*}+k_{i}\right) \iota\left(t_{0}\right) / \lambda_{1}$ as time tends to infinity around the point DFE3. On the contrary, if $v^{*} \leq 1-(v+\gamma+\alpha) / \beta$, then $\lambda_{1} \geq 0$ so that neither the infectious proportion converges to zero nor the vaccinated proportion converges to a constant value as time tends to infinity around the point DFE3.

(d) $k_{s}>0$ and $k_{v} \leq v-\varepsilon_{s} k_{s} /\left(1-\varepsilon_{s}\right)$, then the normalized SISV model only has a DFE point, namely, the point DFE2. Furthermore, if $k_{v}>v-(v+\gamma+\alpha) k_{s} /(\beta-(v+$ $\gamma+\alpha)$ ) then such a DFE point is locally exponentially stable, while if $k_{v}<v-(v+\gamma+\alpha) k_{s} /(\beta-(v+\gamma+\alpha))$, then it is locally exponentially unstable. In the particular case that $k_{v}=v-(v+\gamma+\alpha) k_{s} /(\beta-(v+\gamma+\alpha))$ the dynamics of the infectious proportion is given by $i=0$ in view of the Jacobi matrix $J_{D F E 2}$ in (26). Then, such an infectious proportion is constant in the neighbourhood of the point DFE2. A relevant result is that the point DFE2 is globally stable if $k_{s}>0$ and $k_{v} \in\left(v-(v+\gamma+\alpha) k_{s} /(\beta-(v+\gamma+\alpha)), v-\varepsilon_{s} k_{s} /(1-\right.$ $\left.\varepsilon_{s}\right)$ ] from the following facts: (i) the variables of the normalized SISV epidemic model are bounded, since $s, l, v \in[0,1]$ from the positivity of the original SISV model, (ii) the point DFE2 is the unique equilibrium point of the normalized SISV epidemic model, and (iii) such a point is locally exponentially stable.

(e) $k_{s}>0$ and $k_{v}>v-\varepsilon_{s} k_{s} /\left(1-\varepsilon_{s}\right)$, then the normalized SISV model does not have DFE points too. 
(ii) Note that some of the control gains can take negative values. Moreover, an appropriate choice of such values guarantees the existence of a unique equilibrium point, namely, the point DFE2 defined in (11), while being globally stable. In this sense the gains $k_{s}$ and $k_{v}$ are useful to fix the proportions of susceptible and vaccinated subpopulations when such an equilibrium point is reached. Concretely, a negative value for $k_{v}$ is interesting in order to have a moderate number of vaccinated individuals at such an equilibrium point, which implies a moderate cost in vaccines during the vaccination campaign. The other parameter, i.e., $k_{i}$, can be used to fix the transient behavior of the infection from the starting of the vaccination campaign until the point DFE2 is reached as it is shown by several simulation examples in Section 4.3 of the paper.

(iii) The time evolution of the whole population is given by $\dot{N}(t)=\left(v-\mu-\alpha_{l}(t)\right) N(t) \forall t \geq 0$ from (2) and (4). Then, $\dot{N}(t) \leq(v-\mu) N(t)$ since $\iota(t) \in[0,1] \forall t \geq 0$ from Theorem 2. This implies that $N(t) \leq N\left(t_{0}\right) e^{(v-\mu)\left(t-t_{0}\right)} \forall t \geq t_{0}$ for any arbitrary time instant $t_{0} \geq 0$. On the other hand, the time evolution of the normalized infectious subpopulation in the neighbourhood of the DFE2 point is given by $\iota(t)=$ $\iota\left(t_{1}\right) e^{\lambda_{1}\left(t-t_{1}\right)} \leq \iota_{1} \forall t \geq t_{1}$, where $\iota_{1}=\iota\left(t_{1}\right) \in[0,1], t_{1} \geq 0$ denotes the time instant at which the model state goes in the neighbourhood of the point DFE2, and $\lambda_{1}\left(k_{s}, k_{v}\right)=\beta-$ $(v+\gamma+\alpha)-\beta k_{s} /\left(v-k_{v}+k_{s}\right)<0$ from (26) if the control parameter $\varepsilon_{s}$ and the control gains $k_{s}, k_{i}$, and $k_{v}$ are chosen according to Theorems 3 and 4 so that the DFE2 is the unique equilibrium point of the model and, moreover, globally stable. As a consequence, $I(t)=\iota(t) N(t) \leq I\left(t_{1}\right) e^{\left(\lambda_{1}+v-\mu\right)\left(t-t_{1}\right)} \forall t \geq$ $t_{1}$ under such adjustment of control parameters. This implies that the infectious subpopulation converges exponentially to zero as time goes to infinity if the control gains $k_{s}$ and $k_{v}$ are chosen such that $\lambda_{1}\left(k_{s}, k_{v}\right)<-(v-\mu)$ while the point DFE2 is globally stable. Note that if $v \leq \mu$, i.e., if the birth rate is not larger than the natural death rate, then $\lambda_{1}\left(k_{s}, k_{v}\right)<0 \leq$ $-(v-\mu)$, which implies that that the infectious subpopulation converges exponentially to zero as time goes to infinity for any control parameter $\varepsilon_{s}$ and control gains $k_{s}, k_{i}$, and $k_{v}$ chosen according to Theorems 3 and 4 so that the DFE2 is the unique equilibrium point of the model. Otherwise, i.e., if $v>\mu$, note that $\lambda_{1}\left(0, k_{v}\right)=\beta-(v+\gamma+\alpha)>0$ and $\lim _{k_{s} \longrightarrow \infty}\left\{\lambda_{1}\left(k_{s}, k_{v}\right)\right\}=$ $-(v+\gamma+\alpha)<-(v-\mu)<0$ for any given finite $k_{v}$ and, moreover, $\lambda_{1}\left(k_{s}, k_{v}\right)$ is monotonically decreasing with $k_{s}$ for any $k_{v} \in\left(v-(v+\gamma+\alpha) k_{s} /(\beta-(v+\gamma+\alpha)), v-\varepsilon_{s} k_{s} /\left(1-\varepsilon_{s}\right)\right]$. This implies that $\lambda_{1}\left(k_{s}, k_{v}\right)<\lambda_{\text {crit }}=-(v-\mu) \forall k_{s}>k_{s, \text { crit }}>0$ with $k_{s, c r i t}$ being the value of $k_{s}$ such that $\lambda_{1}\left(k_{s, c r i t}, k_{v}\right)=$ $\lambda_{\text {crit }}$ for a given finite $k_{v} \in\left(v-(v+\gamma+\alpha) k_{s} /(\beta-(v+\right.$ $\left.\gamma+\alpha)), v-\varepsilon_{s} k_{s} /\left(1-\varepsilon_{s}\right)\right]$ from continuity of $\lambda_{1}\left(k_{s}, k_{v}\right)$ with respect to $k_{s}$. In other words, admissible values for the control parameters guaranteeing the exponential convergence to zero of the infectious subpopulation $I(t)$ always exist irrespective of the relation between the birth and natural mortality rates of the host population. Such an appropriate choice of the control parameters implies the eradication of the infection. Furthermore, such an objective can be achieved although the whole population $N(t)$ grows with time because of a higher birth rate related to the natural death rate. This is one of the main results of the current paper and Section 4.2 deals with a numerical example to show the eradication of the infection by applying the proposed vaccination strategy with the aforementioned choice of the control parameters.

\section{Simulation Examples}

4.1. SISV Epidemic Model without Vaccination. Model (1) with a control signal $U(t)=0 \forall t \geq 0$ and an initial condition given by $S(0)=990, I(0)=10$, and $V(0)=0$ is considered. In this way, the vaccination subpopulation is $V(t)=0 \forall t \geq 0$ so that the SISV model is equivalent to a simple SIS model. The values for the parameters $v=2.6301 \times 10^{-5} d^{-1}, \mu=$ $2.4658 \times 10^{-5} d^{-1}, \beta=1.66 d^{-1}, \gamma=0.4545 d^{-1}$, and $\alpha=$ $0.001 d^{-1}$, where $d^{-1}$ means $d a y s^{-1}$, are used to obtain the time evolution of the subpopulations and that of the whole population under the influence of the infectious disease. The proportions of susceptible and infectious subpopulations, i.e., the normalized subpopulations, can be obtained by (4). Also, such subpopulations could be directly obtained using the normalized SISV model (5) with the aforementioned values for the parameters and $u(t)=0 \forall t \geq 0$. The basic reproduction number of this normalized model is $R_{0}=3.6438$ in such a situation so that its DFE point is globally unstable while its EE point is globally asymptotically stable as item (ii) of Remark 1 points out. Such facts are illustrated by means of a simulation example. In this sense, Figure 1 displays the time evolution of the normalized subpopulations of susceptible and infectious individuals in the absence of vaccination. One can see that the proportions of susceptible and infectious subpopulations converge to the values corresponding to the EE point of the normalized SIS model as the theoretical results predict.

Figure 2 shows the time evolution of the susceptible, infectious, and whole populations. One can see that the whole population tends to the extinction because of the dominant effect of the mortality associated with the disease. As a consequence, the application of a vaccination is indispensable in order to eliminate the infection irrespective of the initial conditions or, at least, diminish its effect within the host population and, in this way, achieve the persistence of the host population. Finally, Figure 3 displays the time evolution of the subpopulations and the whole population in the first days of the infection. One can see that the infectious subpopulation quickly increases until it reaches a peak value and then it asymptotically decreases until the extinction of the whole population as Figure 2 shows.

Another example with the same initial condition and the same values for the parameters considered in the previous example, except that $\beta=0.4 d^{-1}$, is analysed. As a result of such a change, the basic reproduction number of the corresponding normalized SISV model without vaccination is $R_{0}=0.878$ so its DFE point is the unique equilibrium point and it is globally asymptotically stable as item (ii) of Remark 1 points out. Figure 4 displays the time evolution of the infectious subpopulation while Figure 5 shows the time evolution of the susceptible subpopulation and the whole population. The infectious disease is eradicated as Figure 4 shows. Moreover, one can deduce that the proportions of 


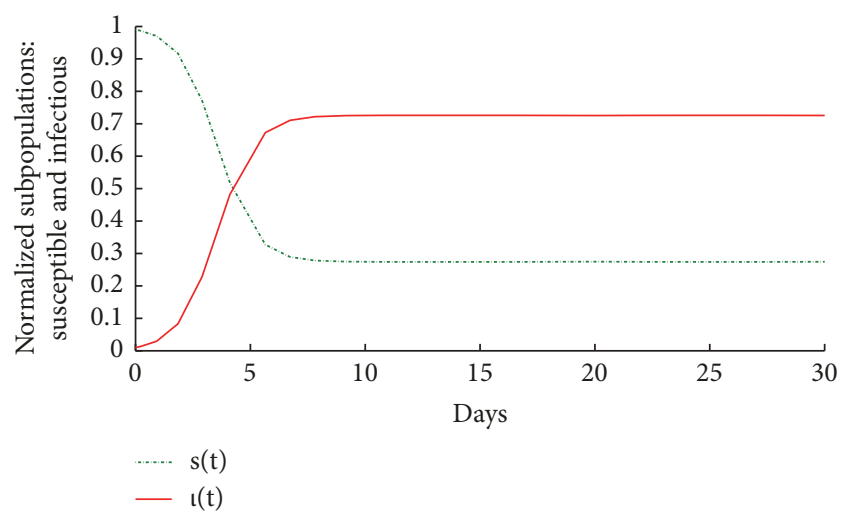

FIGURE 1: Proportions of susceptible and infectious subpopulations in the SISV model without vaccination when the basic reproduction number of its corresponding normalized SISV model is $R_{0}=3.6438$.

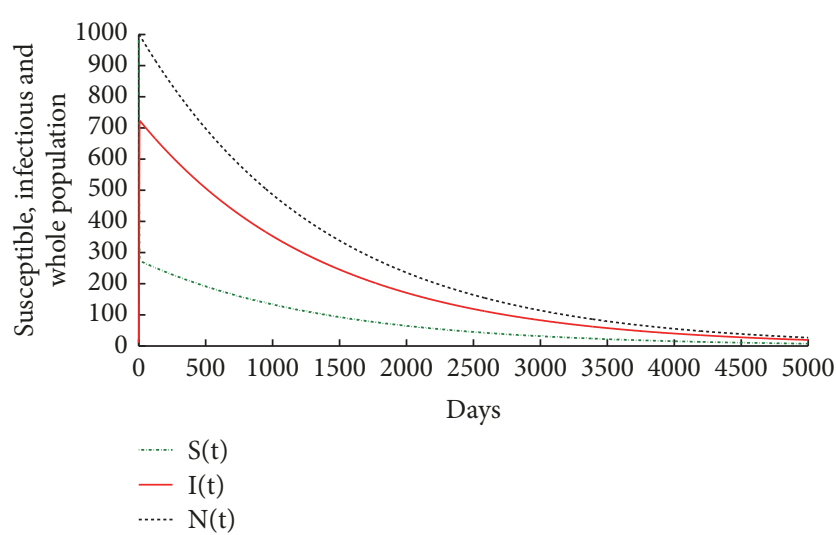

FIGURE 2: Susceptible, infectious, and whole population in the SISV model without vaccination when the basic reproduction number of its corresponding normalized SISV model is $R_{0}=3.6438$.

susceptible and infectious subpopulations converge, respectively, to 1 and 0 , i.e., to the values corresponding to the DFE point as the theoretical results predict. Moreover, one can see in Figure 6 that the whole population increases exponentially once the infectious disease has been eradicated. As a consequence, the application of a vaccination action is not crucial to eliminate the infection although an appropriate vaccination strategy could be used for reducing the time interval until the disease is eradicated.

\subsection{SISV Epidemic Model with Eradication of the Infectious} Disease under a Vaccination Strategy. Model (1) with the same values for the parameters considered in the first example of Section 4.1 is used under the application of a vaccination strategy based on feedback of the model variables as that given in (3). The initial condition is established as $S(0)=990$, $I(0)=10$, and $V(0)=0$. The use of such a vaccination strategy, with an appropriate choice of the free-design control parameters $\varepsilon_{s}, k_{s}, k_{i}$, and $k_{v}$, is crucial to extinguish the disease from the host population since the system without vaccination converges to an EE point as it has been shown in Section 4.1. In this way, a suitable choice of the control

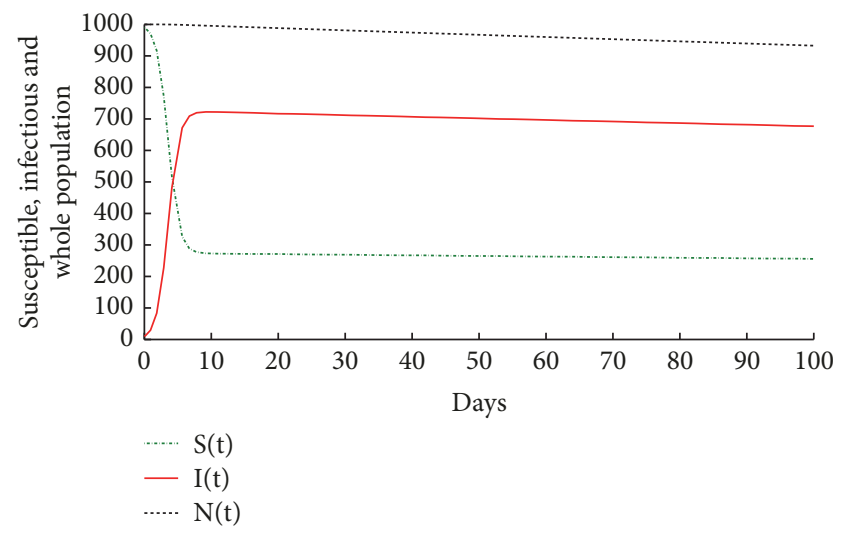

Figure 3: Zoom of Figure 2 in the first days of the transmission of the disease.

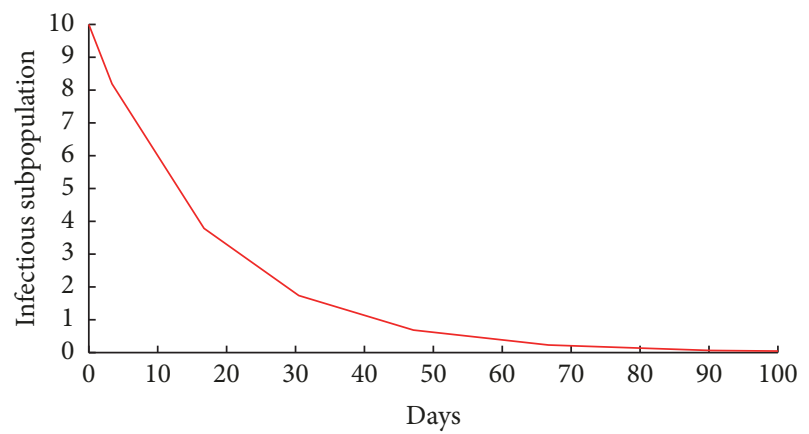

FIgURE 4: Infectious subpopulation in the SISV model without vaccination when the basic reproduction number of its corresponding normalized SISV model is $R_{0}=0.878$.

parameters in order to guarantee the extinction of the disease is $\varepsilon_{s}=0.01, k_{s}=38 v=9.9945 \times 10^{-4}, k_{v}=-13 v=$ $-3.4192 \times 10^{-4}$, and $k_{i}=3000 v=0.0789$. Note that this choice fulfils the conditions (c1), (c2), (c3), (c4), (c5), and (c6)(i) of Theorem 3 so that the normalized SISV model (5) under the control law (6) only has a DFE point, namely, the point DFE2 defined in (11). Moreover, the conditions of Theorem 4 are also satisfied so that such a point is globally stable from the fact that it is locally exponentially stable while the normalized subpopulations are bounded; see item (i) of Remark 5. Such values for the control parameters are used in an example to illustrate the theoretical results predicted in Theorems 3 and 4 and Remark 5. Figure 7 displays the time evolution of the normalized subpopulations of susceptible, infectious, and vaccinated individuals. One can see that the proportions of susceptible, infectious, and vaccinated subpopulations converge to the values corresponding to the point DFE2 of the normalized SISV model, namely, $s_{2}^{*}=$ $0.2692, \iota_{2}^{*}=0$, and $v_{2}^{*}=0.7308$. Moreover, Figure 8 shows the time evolution of the susceptible, infectious, vaccinated, and whole populations. One can see that the number of infectious individuals converges to zero as time tends to infinity and, as a consequence, the disease is eradicated from the host population. Moreover, the infectious population reaches a maximum value at the sixth day from the starting of the 


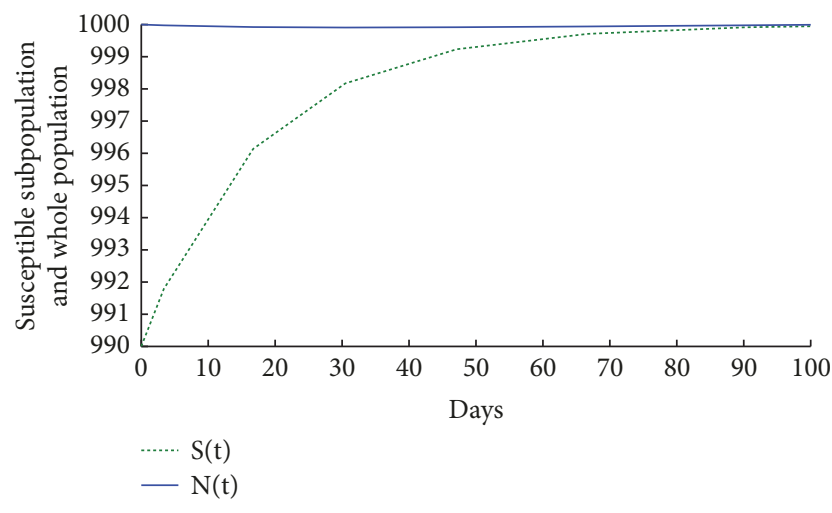

FIGURE 5: Susceptible subpopulation and whole population in the SISV model without vaccination when the basic reproduction number of its corresponding normalized SISV model is $R_{0}=0.878$.

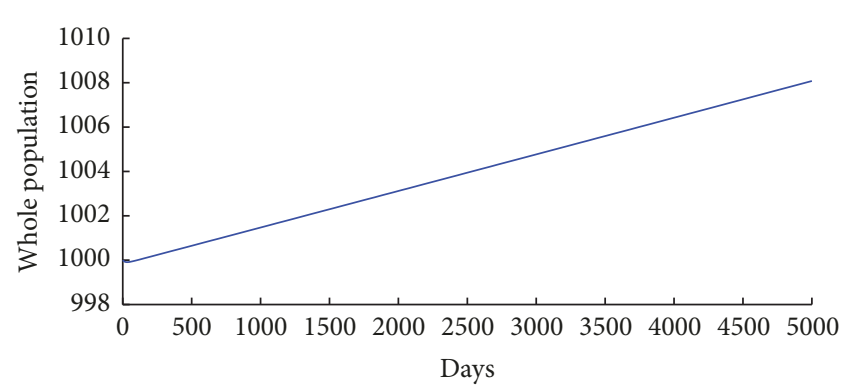

FIGURE 6: Whole population in the SISV model without vaccination when the basic reproduction number of its corresponding normalized SISV model is $R_{0}=0.878$.

infectious disease; namely, $I_{\max }=I(6)=589$. Under practical considerations, the disease can be considered as eradicated when the number of infectious individuals is smaller than 1 since the infection cannot be transmitted when there are not infectious individuals within the host population. Such a fact happens at the day 89 when $I(89)=0.945$ as it has been seen by zooming Figure 8 . Once the infection has been eradicated, from $t=89$, the control signal can be switched off, i.e., $U(t)=0 \forall t>89$, and then the vaccinated subpopulation decreases exponentially to zero since $\dot{V}(t)=-\mu V(t)$ after such a time instant. The decreasing rate is very slow since $\mu<<1$ and then it is not appreciable in Figure 8. The slow increasing of the susceptible subpopulation and the whole population after $t=89$ cannot be seen in Figure 8 for the same reason. A very large duration for the simulation would be necessary to manifest such facts. Also, one can see that the number of vaccinated individuals reaches a maximum value on the day when the vaccination campaign has finished; namely, $V_{\max }=V(89)=763$.

Figure 9 shows the number of vaccines applied during the vaccination campaign. One can see that the injected vaccines reach a peak at the sixth day from the appearance of the infectious disease; namely, $U_{\max }=U(6)=46.7$. Also, the fact that the vaccination effort has to be intensive during the first 30 days reaching a maximum of approximately 47 vaccines at the sixth day is observed. Moreover, the intensity of such an

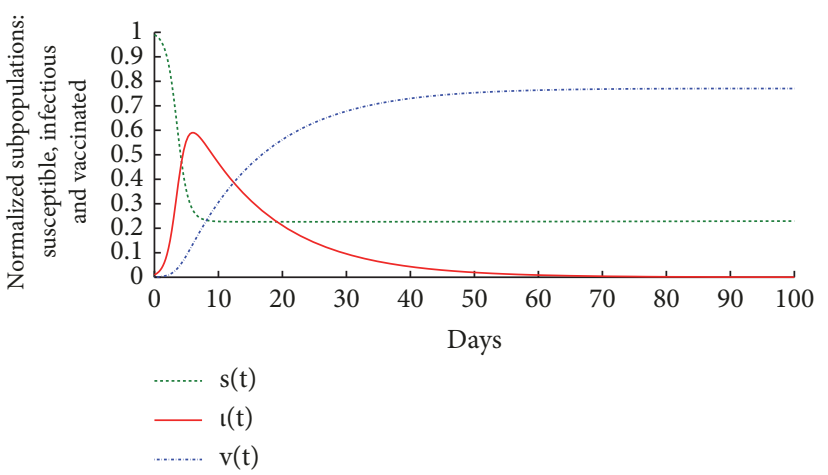

FIGURE 7: Normalized subpopulations of susceptible, infectious, and vaccinated in the SISV model with a vaccination strategy based on the control law (3).

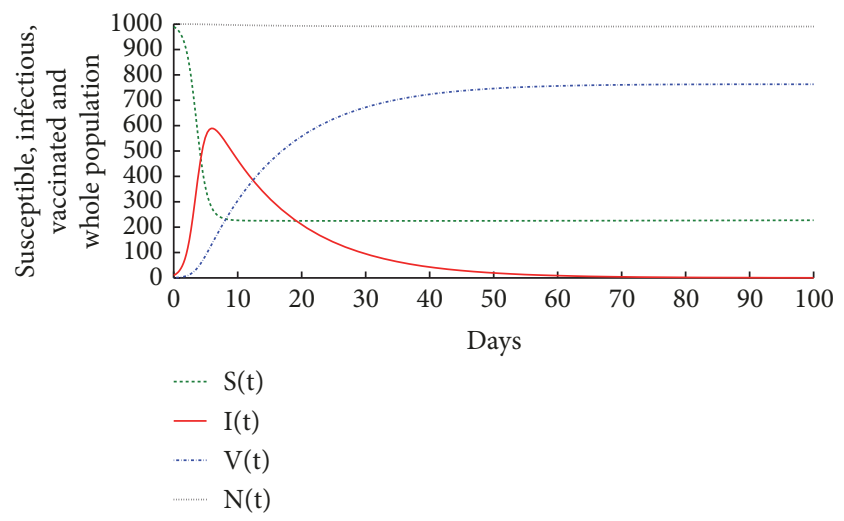

FIGURE 8: Susceptible, infectious, vaccinated, and whole population in the SISV model with a vaccination strategy based on the control law (3).

effort quickly increases until the sixth day and later it slowly decreases until the vaccination campaign finishes. Note that a negative value for $k_{v}$ has been used in this example with the aim of achieving a DFE point, namely, the DFE2 one, in the normalized SISV model with a moderate consumption of vaccines during the vaccination campaign. In this sense, the values for the proportions of susceptible and vaccinated subpopulations once the point DFE2 is reached depend on the values for both $k_{s}$ and $k_{v}$ as the expressions in (11) point out. Concretely, the number of vaccinated individuals at the point DFE2 can be reduced by decreasing the value of $k_{v}$, i.e., by making it more negative while respecting the conditions of Theorem 3 for the unique existence of the point DFE2 and those of Theorem 4 so that such an equilibrium point is locally exponentially stable. In this way, the consumption of vaccines can be reduced. On the other hand, the value of the gain $k_{i}$ can be used to adjust the transient behavior from the starting time instant of the vaccination campaign until the extinction of the disease from the host population as it is illustrated in the following subsection. on the Control Law (3) with Different Values for the Control 


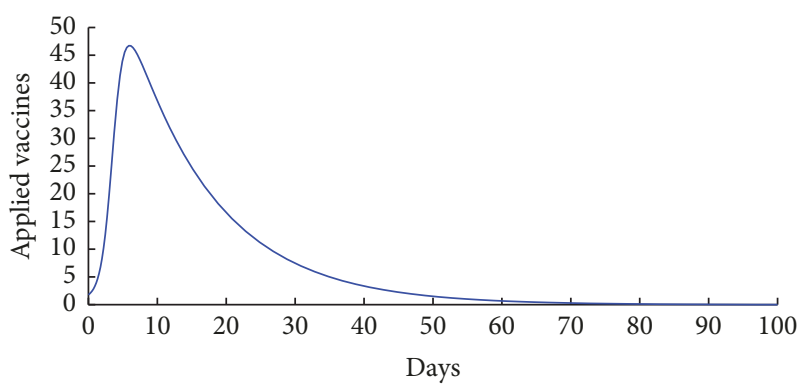

FIGURE 9: Evolution of the vaccines applied during the vaccination campaign.

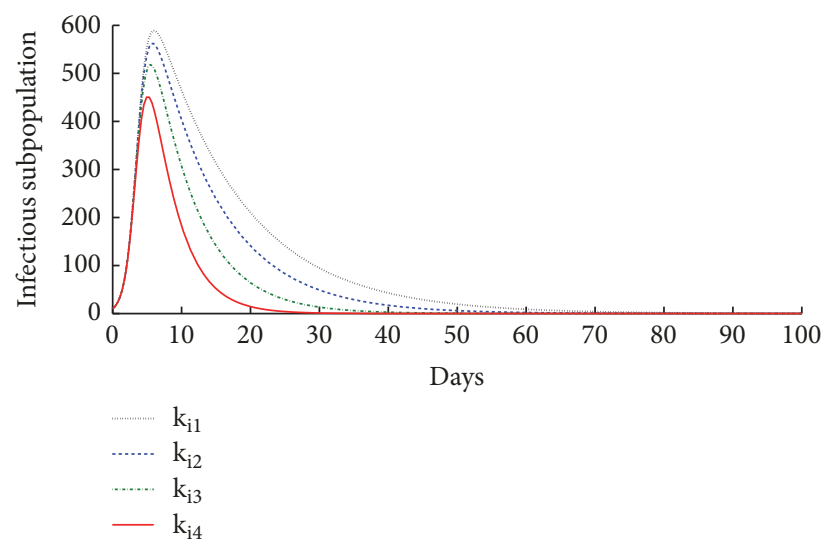

FIGURE 10: Evolution of the infectious subpopulation for different values of $k_{i}$.

Parameter $k_{i}$. The transient behavior of the evolution of the infection from the beginning of the vaccination until its eradication, whenever the control parameters are appropriately chosen in order to achieve such an objective as it has been pointed out in Remark 5, depends on the values of such parameters. The maximum vaccinated individuals $V_{\max }$, the maximum in the number of vaccines $U_{\max }$, the maximum of the infectious individuals $I_{\max }$, the instants when such maximum values occur, and the duration of the vaccination campaign $\Delta t_{v a c}$ are some specifications used to characterise this transient behavior. The dependence of such specifications on the value of the control gain $k_{i}$ is shown in this example. Concretely, $\varepsilon_{s}=0.01, k_{s}=38 v=9.9945 \times 10^{-4}$, and $k_{v}=$ $-13 v=-3.4192 \times 10^{-4}$ are maintained as in Section 4.2 while several values for $k_{i}$ are considered, namely, $k_{i 1}=3000 \mathrm{v}=$ $0.0789, k_{i 2}=4000 v=0.1052, k_{i 3}=6000 v=0.1578$, and $k_{i 4}=10000 v=0.263$ in four simulations with the same values for the parameters of the SISV model and the same initial condition as those used in Section 4.2. Note that the chosen values for $\varepsilon_{s}, k_{s}$, and $k_{v}$, together with any of the values for $k_{i}$, i.e., $k_{i 1}, k_{i 2}, k_{i 3}$, or $k_{i 4}$, fulfil the conditions (c1), (c2), (c3), (c4), (c5), and (c6.i) of Theorem 3 so that the normalized SISV model (5) under the control law (6) only has a DFE point, namely, the point DFE2 defined in (11). Moreover, the conditions of Theorem 4 are also satisfied so that such a point is globally stable from the facts that it is locally exponentially stable while the normalized subpopulations are

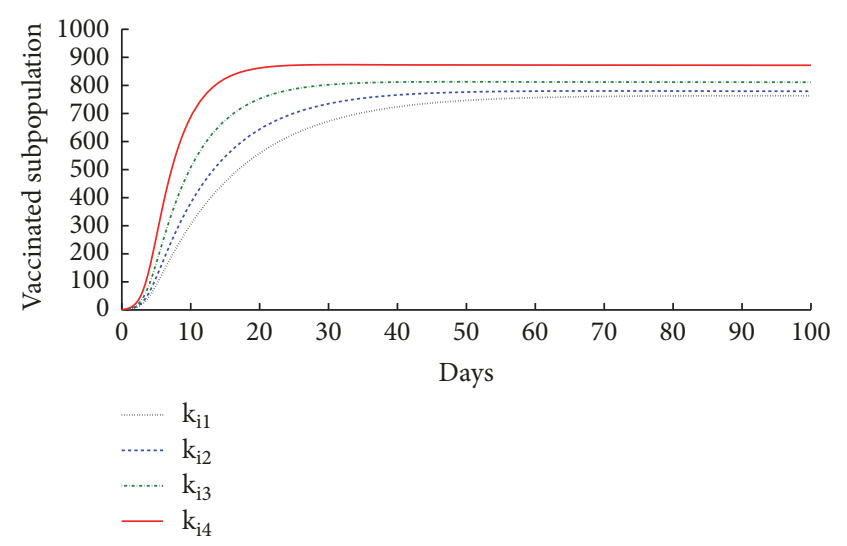

FIGURE 11: Evolution of the vaccinated subpopulation for different values of $k_{i}$.

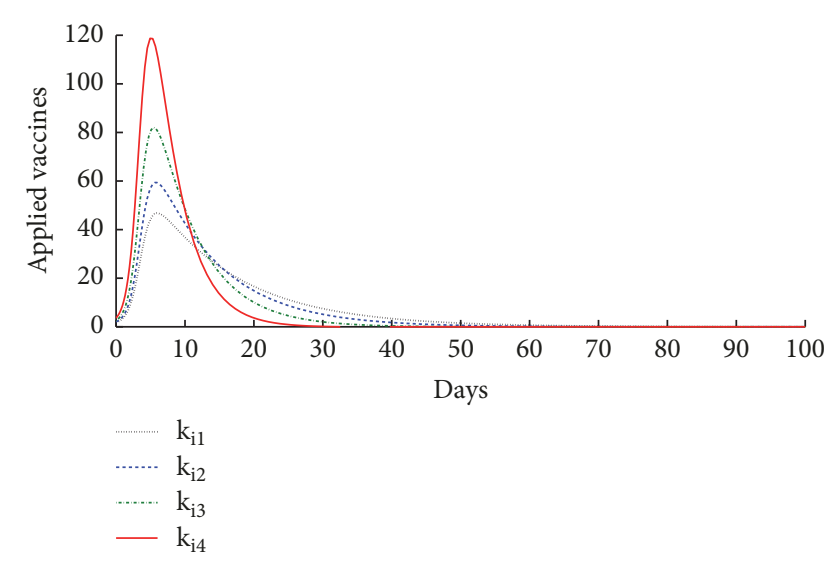

FIGURE 12: Evolution of the vaccines applied during the vaccination campaign for different values of $k_{i}$.

bounded; see item (i) of Remark 5. Indeed, the first value $k_{i 1}$ coincides with that used in Section 4.2 since such a simulation example is used as a reference in order to show the differences in the performance of the vaccination campaign by increasing the value of the control parameter $k_{i}$. Figure 10 displays the time evolution of the infectious subpopulation during the vaccination campaign. One can see that the peak in the number of infectious decreases as the value of the control parameter $k_{i}$ increases. Moreover, the day on which the number of infectious individuals is smaller than 1 , and then the infection can be considered eradicated, decreases as $k_{i}$ increases. Also, such a day can be considered as the end of the vaccination campaign.

Figure 11 shows the time evolution of the vaccinated subpopulation during the vaccination campaign. The maximum value reached for the vaccinated subpopulation increases as $k_{i}$ increases. Such a maximum is achieved at the last day of the vaccination campaign. As a consequence, the number of applied vaccines increases as $k_{i}$ increases.

The application of the vaccines to the susceptible subpopulation is distributed through time as Figure 12 shows. One can see that the peak in the vaccines increases as $k_{i}$ increases while the day on which such a peak is achieved is 


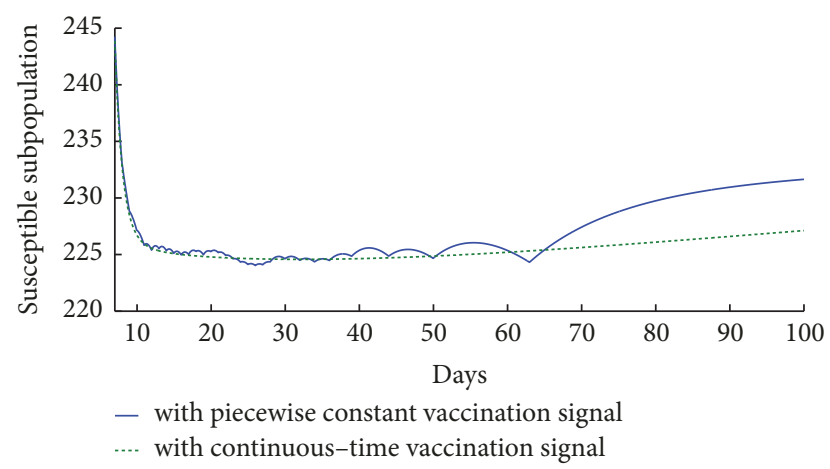

FIGURE 13: Susceptible subpopulation in the SISV model with two different vaccination signals: (i) piecewise constant signal taking integer values and (ii) continuous-time signal.

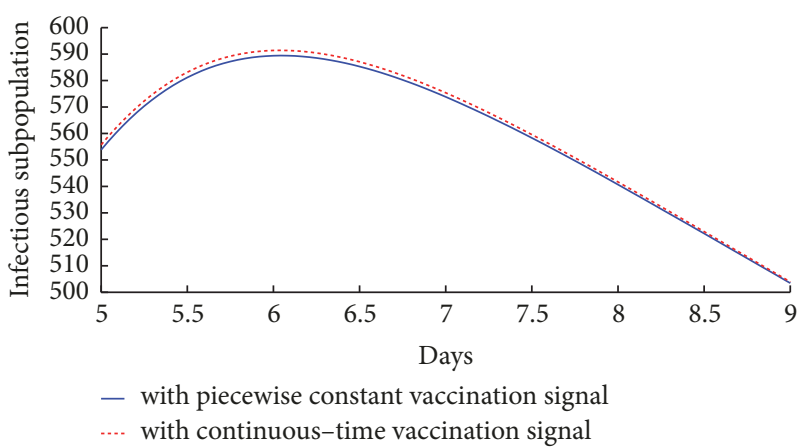

FIGURE 14: Infectious subpopulation in the SISV model with two different vaccination signals: (i) piecewise constant signal taking integer values and (ii) continuous-time signal.

irrespective of $k_{i}$ for the considered values, namely, the sixth day for all cases. Note that such an increment in the peak of the required vaccines is due to two reasons; namely, the number of vaccinated subpopulation increases as $k_{i}$ increases while, on the other hand, the duration of the vaccination campaign decreases as $k_{i}$ increases. In other words, a larger number of vaccines have to be applied in a shorter vaccination campaign as $k_{i}$ increases. Moreover, the number of required vaccines during the vaccination campaign can be adjusted by the control gain $k_{v}$ as it has been pointed out in Section 4.2. Concretely, the lower the value of $k_{v}$, the lower the quantity of required vaccines. As a consequence, a trade-off between the number of available vaccines and the duration of the vaccination campaign has to be taken into account to choose both control parameters $k_{i}$ and $k_{v}$ while guaranteeing that the control parameters are chosen so that the point DFE2 is unique and locally asymptotically stable. The values of the aforementioned specifications for the different values considered for the parameter $k_{i}$ are detailed in Table 2 .

4.4. SISV Epidemic Model with a Piecewise Constant Vaccination Function Taking Integer Values. The same values used in Section 4.2 for the parameters of the SISV model and for the vaccination control law (3) are considered in this simulation example. Also, the same initial condition is used.

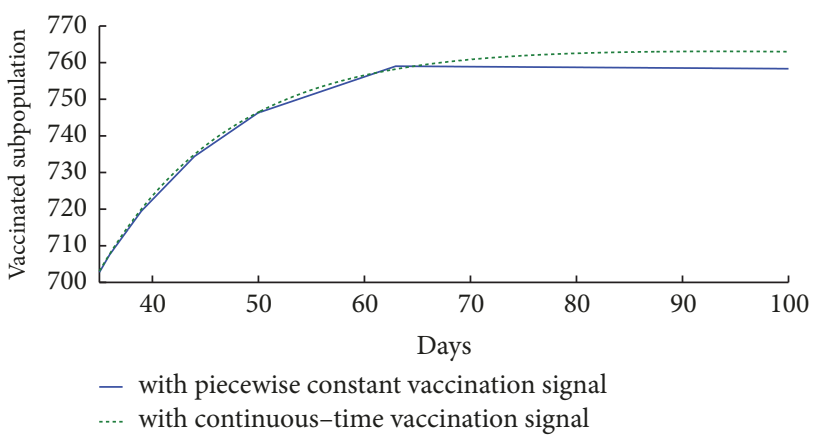

FIGURE 15: Vaccinated subpopulation in the SISV model with two different vaccination signals: (i) piecewise constant signal taking integer values and (ii) continuous-time signal.

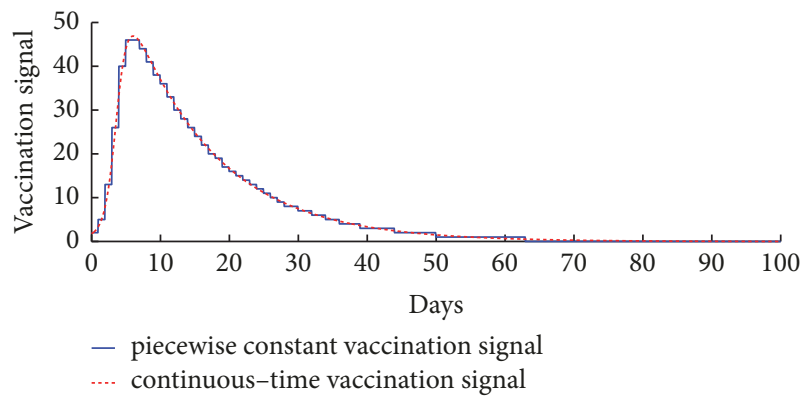

FIGURE 16: Evolution of the vaccination signals: (i) piecewise constant signal taking integer values and (ii) continuous-time signal.

The difference with respect to Section 4.2 is that the control signal $U(t)$ can only take nonnegative integer values during the vaccination campaign in this example. Such a restriction is logic from a practical viewpoint since $U(t)$ denotes the number of injected vaccines. The values for $U(t)$ at each day in the current example are taken from averaging the values in Figure 9 during such a day and taking the integer closest to such an average value. The time evolution of the susceptible subpopulation in this example compared with the time evolution of such a subpopulation in the example of Section 4.2 is displayed in Figure 13. Figure 14 shows the comparison between the time evolution of the infectious subpopulations in the current example and that of Section 4.2 while Figure 15 displays the comparison of the time evolution of the vaccinated subpopulations in both examples. Such figures mainly show the evolution within the time interval when there is an appreciable difference between the obtained results with these two different control strategies. Finally, Figure 16 displays the time evolution of the two different control signals, namely, the piecewise constant vaccination signal taking integer values used in the current example and the continuous-time one used in that of Section 4.2.

One can see that the differences in Figures 13, 14, and 15 are very small. Such a fact implies that a practical vaccination campaign can be carried out by building a piecewise constant control signal taking nonnegative integer values from the control signal (3) as it has been done in the current example. However, the current example has been performed offline 
TABLE 2: Specifications of the vaccination campaign for the different values of $k_{i}$.

\begin{tabular}{lccccc}
\hline$k_{i}$ & $I_{\max }$ & $V_{\max }$ & $U_{\max }($ vaccines $)$ & $\Delta t_{\text {vac }}($ days $)$ & $t_{I_{\max }} \approx t_{U_{\max }}($ days $)$ \\
\hline $3000 v$ & 589 & 763 & 46.7 & 69 & 6 \\
$4000 v$ & 582 & 780 & 60 & 68 & 6 \\
$6000 v$ & 518 & 813 & 82 & 47 & 5.5 \\
$10000 v$ & 450 & 874 & 119 & 31 & 5 \\
\hline
\end{tabular}

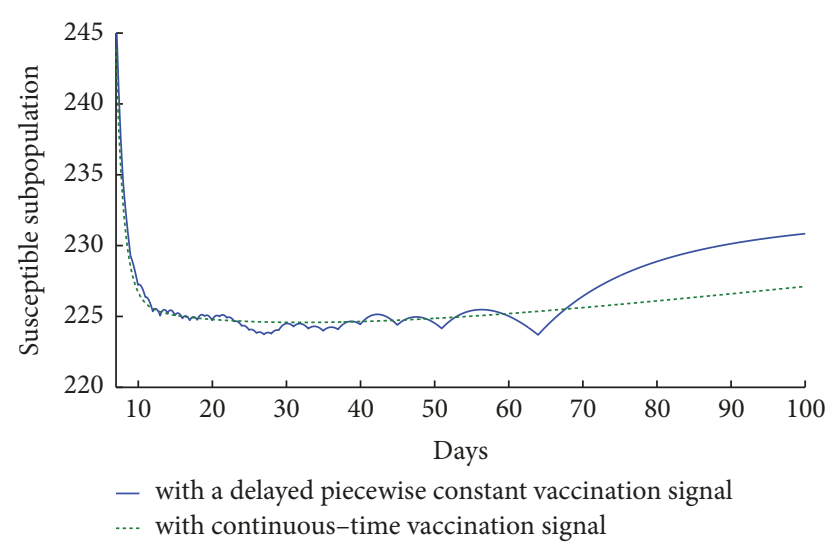

FIGURE 17: Susceptible subpopulation in the SISV model with two different vaccination signals: (i) a delayed piecewise constant signal taking integer values and (ii) continuous-time signal.

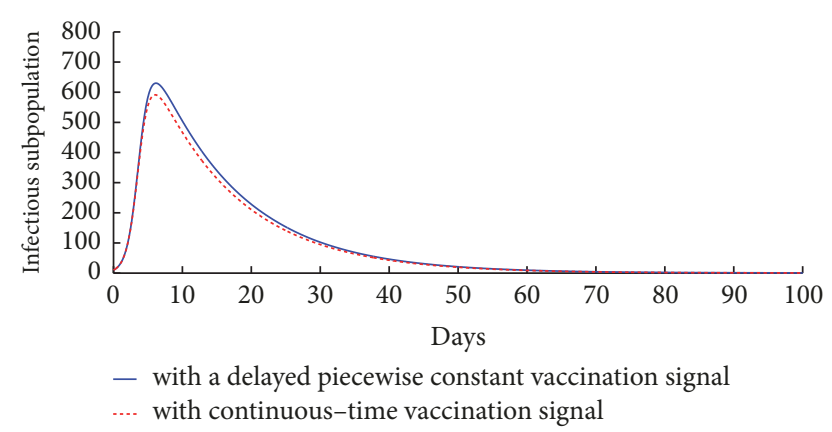

FIGURE 18: Infectious subpopulation in the SISV model with two different vaccination signals: (i) a delayed piecewise constant signal taking integer values and (ii) continuous-time signal.

since the piecewise control signal has been built with the vaccination control law values stored after simulating the example of Section 4.2.

The implementation of a piecewise control strategy online can be done with a delay of one day by applying each day the number of vaccines corresponding to the average of the values of the control signal stored in the previous day. Figures 17-20 display the results obtained by using such a strategy compared with those corresponding to the example of Section 4.2. The main difference in the online application of the vaccination signal with respect to the offline one is appreciable in the time evolution of the infectious subpopulation. One can see that the peak achieved in the infectious subpopulation under the application of the one day delayed piecewise constant vaccination is larger than that

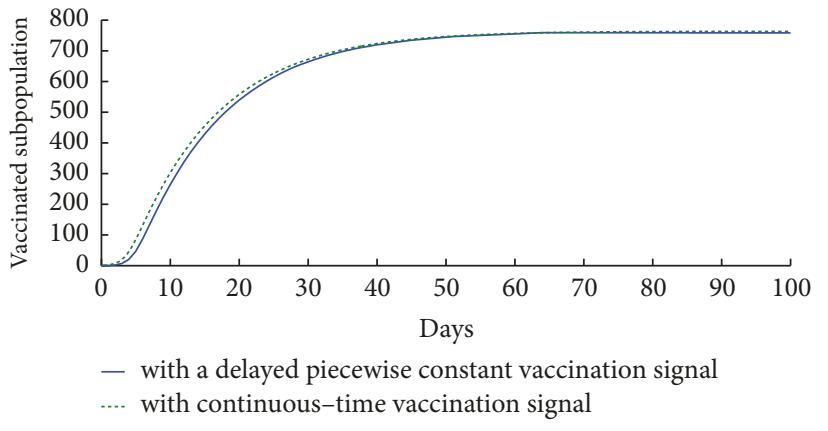

FIGURE 19: Vaccinated subpopulation in the SISV model with two different vaccination signals: (i) a delayed piecewise constant signal taking integer values and (ii) continuous-time signal.

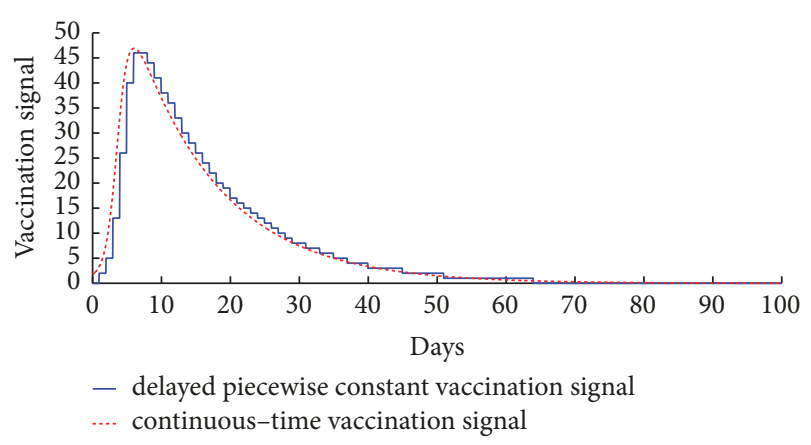

FIGURE 20: Evolution of the vaccination signals: (i) a delayed piecewise constant signal taking integer values and (ii) continuoustime signal.

achieved with a piecewise constant vaccination without delay. However, such a delay does not inhibit the eradication of the disease but the transient behavior until the total extinction of the disease is slightly worse.

\subsection{SISV Epidemic Model under a Vaccination with Uncertain-} ties in the Synthesis of the Control Signal. The exact measure of the amount of individuals in each category of the model at each time instant is unviable in a real situation. This fact impedes the synthesis of the proposed vaccination control signal by means of (3). Moreover, the amount of individuals in each category as well as the quantity of vaccines to be applied has to be an integer number. An alternative way for synthesizing a vaccination signal taking into account such constraints is considered as follows. First, the quantity of individuals in each category is measured one time per day although the model runs in continuous-time. A random 
number is added to each measure to take into account the fact that such measures can be imprecise; i.e., they are subject to the presence of uncertainties, as it may happen in a real situation. The integer that is closest to the measure with uncertainty of each subpopulation is used to synthesize the control law in order to calculate the integer number of vaccines to be applied at each day. Such available measures of each subpopulation at each day, namely, $S_{u}(n), I_{u}(n)$, and $V_{u}(n)$, can be expressed as

$$
\begin{aligned}
S_{u}(n) & =\operatorname{round}\left(\max \left\{0,\left(1+\delta_{S}(n)\right) S(n)\right\}\right) ; \\
I_{u}(n) & =\operatorname{round}\left(\max \left\{0,\left(1+\delta_{I}(n)\right) I(n)\right\}\right) \\
V_{u}(n) & =\operatorname{round}\left(\max \left\{0,\left(1+\delta_{V}(n)\right) V(n)\right\}\right) ; \\
N_{u}(n) & =S_{u}(n)+I_{u}(n)+V_{u}(n)
\end{aligned}
$$

with $n$ being a nonnegative integer number. The terms $S(n)$, $I(n)$, and $V(n)$ denote the measures, without uncertainties, of susceptible, infectious, and vaccinated subpopulations at each day, respectively. The functions $\delta_{S}(n), \delta_{I}(n)$, and $\delta_{V}(n)$ provide random numbers used to take into account the presence of uncertainties in the measures. Such numbers are within the interval $[-0.1,0.1]$ so that the discrepancy between each measure and the real value of the corresponding subpopulation is of $10 \%$ at the most. Note that the function round $\{x\}$, which returns the integer that is closest to the real number $x$, and the function $\max \{0, x\}$ are used to guarantee that the measures with uncertainties are nonnegative integers. The sum $N_{u}(n)=S_{u}(n)+I_{u}(n)+V_{u}(n)$ is the measure of the whole population with uncertainty. Such measures with uncertainties are available to synthesize the control signal given by

$$
U(t)=\operatorname{round}\{U(n)\} \quad \text { for } n T \leq t<(n+1) T
$$

where $T=1$ day, since the subpopulation measures are done once per day so that the control signal $U(t)$ is a piecewise constant function, and

$$
\begin{aligned}
& U(n) \\
& = \begin{cases}\max \left\{0, k_{s} S_{u}(n)+k_{i} I_{u}(n)+k_{v} V_{u}(n)\right\} & \text { if } S_{u}(n) \geq \varepsilon_{s} N_{u}(n) \\
0 & \text { otherwise. }\end{cases}
\end{aligned}
$$

Note that the vaccination signal $U(t)$, which denotes the number of vaccines to be applied within each day, is calculated by using the measures with uncertainties of the subpopulations in the previous day.

A simulation example is carried out in this realistic situation, i.e., with a vaccination campaign supervised by the control law (31)-(32) with the same values for the control parameters, i.e., $\varepsilon_{s}=0.01, k_{s}=38 v, k_{v}=-13 v$, and $k_{i}=3000 v$, as those used in the example of Section 4.2. The derived results are compared with those obtained in the example of Section 4.2 where the basic control law (3) was used. Figures 21, 22, and 23 show, respectively, the comparison between the time evolution of the susceptible, infectious, and vaccinated subpopulations in the current example with those of Section 4.2 while Figure 24 displays the time evolution

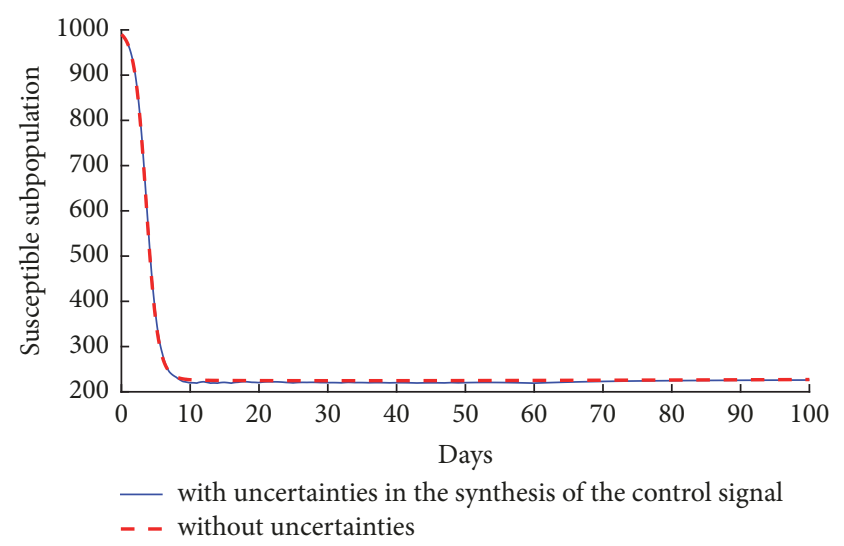

FIGURE 21: Susceptible subpopulations with (i) the vaccination strategy designed if there are uncertainties in the measures of the subpopulations and (ii) the basic vaccination strategy without uncertainties.

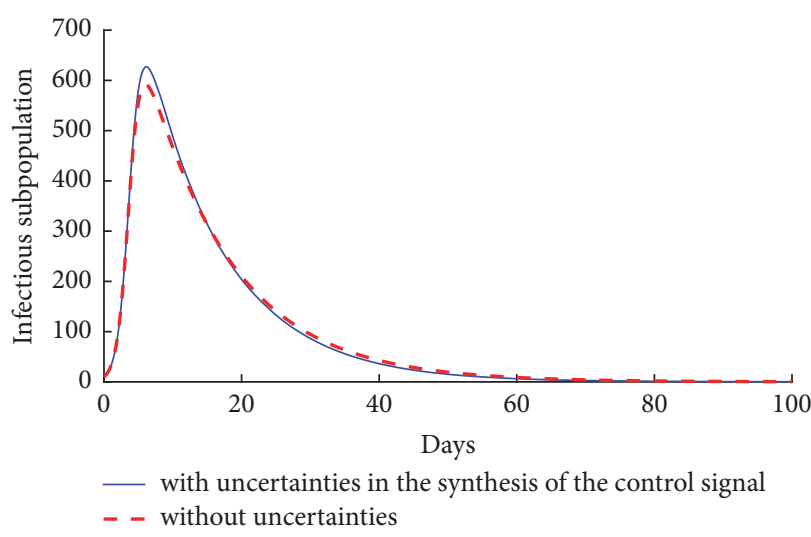

FIGURE 22: Infectious subpopulations with (i) the vaccination strategy designed if there are uncertainties in the measures of the subpopulations and (ii) the basic vaccination strategy without uncertainties.

of the two different control signals, namely, the piecewise constant vaccination signal taking integer values used in the current example and the continuous-time one used in that of Section 4.2.

One can see that the results obtained in the current example are quite similar to those obtained in that of Section 4.2. This fact implies that a vaccination strategy supervised by the control signal (31)-(32) leads to the eradication of the infectious diseases in spite of eventual presence of small uncertainties in the measures of the subpopulations. Finally, Figure 25 compares the time evolution of the measures used to synthesize the control signal by means of (31)-(32) with the real values of the subpopulations and whole population at each time instant. This figure highlights the presence of discrepancies between the real values of the subpopulations and those used to synthesize the control signal during all the simulation time. In summary, a suitable adjustment of the control gains achieves the eradication of the infectious disease although the measures of the subpopulations needed to synthesize the control signal are subject to uncertainties. 


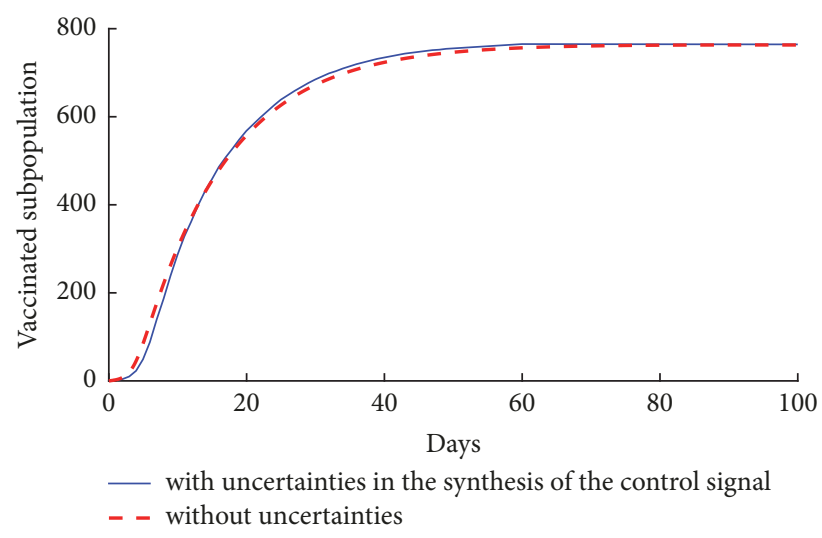

FIGURE 23: Vaccinated subpopulations with (i) the vaccination strategy designed if there are uncertainties in the measures of the subpopulations and (ii) the basic vaccination strategy without uncertainties.

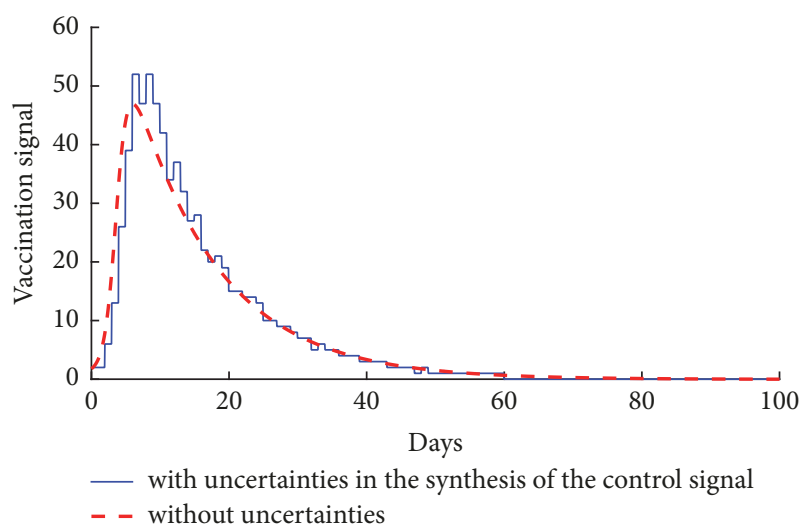

Figure 24: Applied vaccines with (i) the vaccination strategy designed if there are uncertainties in the measures of the subpopulations and (ii) the basic vaccination strategy without uncertainties.

In this sense, the designed vaccination strategy with the appropriate control gains is robust against the presence of uncertainties in the measures used to synthesize the control signal.

\section{Conclusions}

A vaccination strategy based on the state feedback control theory has been designed in order to eradicate the persistence of an infectious disease within a host population. Such a vaccination is applied in a SISV epidemic model. The positivity property of such a model is analysed as well as the existence and stability of the equilibrium points of their corresponding normalized model under such a vaccination strategy. The vaccination provides four free-design control parameters. Three of them are the gains to synthesize the state feedback control signal. The eradication of the infection is achieved by adjusting the control parameters to appropriate values such that there are not EE points in the normalized SISV model while a globally exponentially stable DFE is its unique equilibrium point. In such a case, the control gains

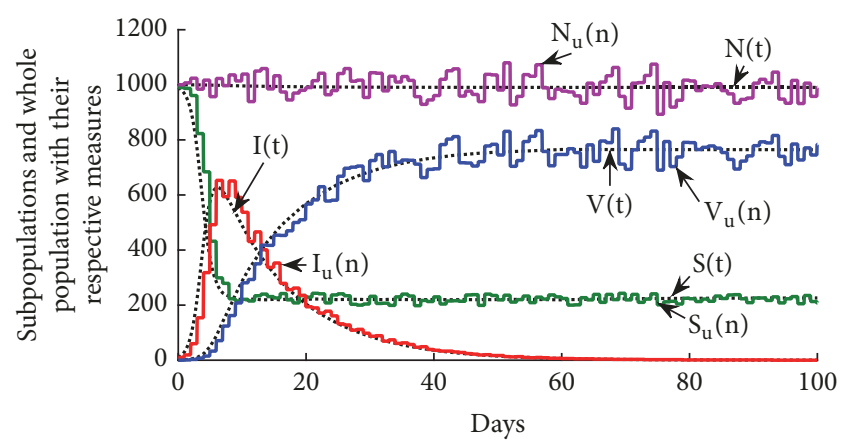

FIgURE 25: Subpopulations measures used to synthesize the vaccination control signal in comparison with the real time evolution of such subpopulations and whole population.

multiplying to the susceptible and vaccinated subpopulations, respectively, $k_{s}$ and $k_{v}$, can be adjusted to fix the proportion of susceptible and vaccinated subpopulation to desired values once such a DFE point is reached. Furthermore, the number of vaccines used in the vaccination campaign decreases if the value for the gain $k_{v}$ decreases and, in this context, it is worth pointing out that such a gain can take negative values. Moreover, the gain acting on the infectious subpopulation, namely $k_{i}$, can be adjusted to appropriately shape the transient behavior in the evolution of the disease until its total eradication. Such a transient behavior is characterized by three main specifications: (i) duration of the vaccination campaign, (ii) the peak value of the infectious subpopulation, and (iii) the number of vaccines used within the vaccination period. A trade-off between the number of vaccines and the duration of the vaccination campaign has to be taken into account to choose the control gains. In this context, the number of vaccines may be used to fix the value of $k_{v}$, and the duration of the vaccination campaign can be prefixed by the value of the gain $k_{i}$. Note that such adjustments for the gains $k_{i}$ and $k_{v}$ have to be compatible with the conditions guaranteeing the existence of a globally stable DFE point in the normalized SISV model. Some simulation examples emphasize the theoretic results presented in the paper. Other ones point out alternative ways of implementing a vaccination campaign based on the proposed state feedback control technique in a real situation with the presence of uncertainties in the measures of the subpopulations needed to synthesize the control law.

An extension of the current paper might be the design of other types of control signals, as, for instance, impulsive vaccination strategies adjusting the gain of the impulses and/or the interval between consecutive impulses. Another extension relies on the consideration of delays in the SISV model since, for instance, the effect of a vaccine in the vaccinated individuals cannot be immediate but it could be subject to a certain delay.

\section{Data Availability}

The underlying data to support this study are included within the references. 


\section{Conflicts of Interest}

The authors declare that they have no conflicts of interest.

\section{Authors' Contributions}

All the authors contribute equally to all the parts of the manuscript.

\section{Acknowledgments}

This research is supported by the Spanish Government through Grant DPI2015-64766-R and by UPV/EHU through Grant PGC 17/33.

\section{References}

[1] M. J. Keeling and P. Rohani, Modeling Infectious Diseases in Humans and Animals, Princeton University Press, Princeton, NJ, USA, 2008.

[2] O. Diekmann and J. A. P. Heesterbeek, Mathematical Epidemiology of Infectious Diseases, Model Building, Analysis and Interpretation, John Wiley \& Sons, 2000.

[3] S. Alonso-Quesada, M. De la Sen, A. Ibeas, and R. Nistal, "A vaccination strategy based on linearization control techniques for fighting against epidemic diseases propagation," Advances in Difference Equations, 2013:364, 18 pages, 2013.

[4] G. P. Sahu and J. Dhar, "Dynamics of an SEQIHRS epidemic model with media coverage, quarantine and isolation in a community with pre-existing immunity," Journal of Mathematical Analysis and Applications, vol. 421, no. 2, pp. 1651-1672, 2015.

[5] G. Chowell, C. E. Ammon, N. W. Hengartner, and J. M. Hyman, "Transmission dynamics of the great influenza pandemic of 1918 in Geneva, Switzerland: assessing the effects of hypothetical interventions," Journal of Theoretical Biology, vol. 241, no. 2, pp. 193-204, 2006.

[6] A. R. Carvalho and C. M. Pinto, "Emergence of drug-resistance in HIV dynamics under distinct HAART regimes," Communications in Nonlinear Science and Numerical Simulation, vol. 30, no. 1-3, pp. 207-226, 2016.

[7] X. Liu and P. Stechlinski, "Application of control strategies to a seasonal model of chikungunya disease," Applied Mathematical Modelling: Simulation and Computation for Engineering and Environmental Systems, vol. 39, no. 12, pp. 3194-3220, 2015.

[8] J. Li, Z. Ma, and Y. Zhou, "Global analysis of SIS epidemic model with a simple vaccination and multiple endemic equilibria," Acta Mathematica Scientia B, vol. 26, no. 1, pp. 83-93, 2006.

[9] X. B. Liu and L. J. Yang, "Stability analysis of an SEIQV epidemic model with saturated incidence rate," Nonlinear Analysis: Real World Applications, vol. 13, no. 6, pp. 2671-2679, 2012.

[10] X.-Z. Li, W.-S. Li, and M. Ghosh, "Stability and bifurcation of an SIR epidemic model with nonlinear incidence and treatment," Applied Mathematics and Computation, vol. 210, no. 1, pp. 141150, 2009.

[11] B. Buonomo, A. d'Onofrio, and D. Lacitignola, "Global stability of an SIR epidemic model with information dependent vaccination," Mathematical Biosciences, vol. 216, no. 1, pp. 9-16, 2008.

[12] A. Ibeas, M. de la Sen, and S. Alonso-Quesada, "Adaptive control of SEIR discrete-time epidemic models," in Proceedings of the 10th international conference on mathematical problems in engineering, aerospace and sciences: ICNPAA '14, pp. 37-46, Narvik, Norway.

[13] Y. Xiao, X. Xu, and S. Tang, "Sliding mode control of outbreaks of emerging infectious diseases," Bulletin of Mathematical Biology, vol. 74, no. 10, pp. 2403-2422, 2012.

[14] G. Zaman, Y. H. Kang, G. Cho, and I. H. Jung, "Optimal strategy of vaccination \& treatment in an SIR epidemic model," Mathematics and Computers in Simulation, vol. 136, pp. 63-77, 2017.

[15] P. Yongzhen, S. Li, C. Li, and S. Chen, "The effect of constant and pulse vaccination on an SIR epidemic model with infectious period," Applied Mathematical Modelling, vol. 35, no. 8, pp. 3866-3878, 2011.

[16] J. Hou and Z. Teng, "Continuous and impulsive vaccination of SEIR epidemic models with saturation incidence rates," Mathematics and Computers in Simulation, vol. 79, no. 10, pp. 3038-3054, 2009.

[17] Z. Yang and H. Jia, "Epidemic dynamics model with delay and impulsive vaccination control base on variable population," Mathematical Methods in the Applied Sciences, vol. 34, no. 15, pp. 1822-1832, 2011.

[18] S. Alonso-Quesada, M. De la Sen, and A. Ibeas, "On the discretization and control of an SEIR epidemic model with a periodic impulsive vaccination," Communications in Nonlinear Science and Numerical Simulation, vol. 42, pp. 247-274, 2017.

[19] C. Vargas-De-Leon, "On the global stability of SIS, SIR and SIRS epidemic models with standard incidence," Chaos, Solitons \& Fractals, 2011.

[20] X. Z. Meng, S. N. Zhao, T. Feng, and T. H. Zhang, "Dynamics of a novel nonlinear stochastic SIS epidemic model with double epidemic hypothesis," Journal of Mathematical Analysis and Applications, vol. 433, no. 1, pp. 227-242, 2016.

[21] M. Kretzschmar and J. C. Heijne, "Pair formation models for sexually transmitted infections: A primer," Infectious Disease Modelling, vol. 2, no. 3, pp. 368-378, 2017.

[22] C. Sun and Y. Hsieh, "Global analysis of an SEIR model with varying population size and vaccination," Applied Mathematical Modelling, vol. 34, no. 10, pp. 2685-2697, 2010.

[23] J. Zhang, J. Jia, and X. Song, "Analysis of an SEIR epidemic model with saturated incidence and saturated treatment function," The Scientific World Journal, 2014.

[24] J. Mena-Lorca and H. W. Hethcote, "Dynamic models of infectious diseases as regulators of population sizes," Journal of Mathematical Biology, vol. 30, no. 7, pp. 693-716, 1992.

[25] Y. Cai, Y. Kang, and W. Wang, "A stochastic SIRS epidemic model with nonlinear incidence rate," Applied Mathematics and Computation, vol. 305, pp. 221-240, 2017.

[26] C. M. Kribs-Zaleta and J. X. Velasco-Hernández, "A simple vaccination model with multiple endemic states," Mathematical Biosciences, vol. 164, no. 2, pp. 183-201, 2000.

[27] X. Liu, Y. Takeuchi, and S. Iwami, "SVIR epidemic models with vaccination strategies," Journal of Theoretical Biology, vol. 253, no. 1, pp. 1-11, 2008.

[28] X. C. Duan, S. L. Yuan, Z. P. Qiu, and J. L. Ma, "Global stability of an SVEIR epidemic model with ages of vaccination and latency," Computers \& Mathematics with Applications, vol. 68, no. 3, pp. 288-308, 2014.

[29] A. d'Onofrio, P. Manfredi, and E. Salinelli, "Vaccinating behaviour, information, and the dynamics of SIR vaccine preventable diseases," Theoretical Population Biology, vol. 71, no. 3, pp. 301-317, 2007. 
[30] A. Pugliese, "Population models for diseases with no recovery," Journal of Mathematical Biology, vol. 28, no. 1, pp. 65-82, 1990.

[31] S. Sharma and G. P. Samanta, "Stability analysis and optimal control of an epidemic model with vaccination," International Journal of Biomathematics, vol. 8, no. 3, Article ID 1550030, 28 pages, 2015.

[32] F. Brauer, C. Castillo-Chavez, A. Mubayi, and S. Towers, "Some models for epidemics of vector-transmitted diseases," Infectious Disease Modelling, vol. 1, no. 1, pp. 79-87, 2016.

[33] Q. Hou and T. Wang, "Global stability and a comparison of SVEIP and delayed SVIP epidemic models with indirect transmission," Communications in Nonlinear Science and Numerical Simulation, vol. 43, pp. 271-281, 2017.

[34] S. A. Pedro, H. E. Tonnang, and S. Abelman, "Uncertainty and sensitivity analysis of a Rift Valley fever model," Applied Mathematics and Computation, vol. 279, pp. 170-186, 2016.

[35] L. Shaikhet and A. Korobeinikov, "Stability of a stochastic model for HIV-1 dynamics within a host," Applicable Analysis: An International Journal, vol. 95, no. 6, pp. 1228-1238, 2016. 


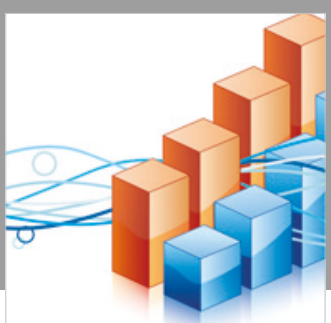

Advances in

Operations Research

\section{-n-m}
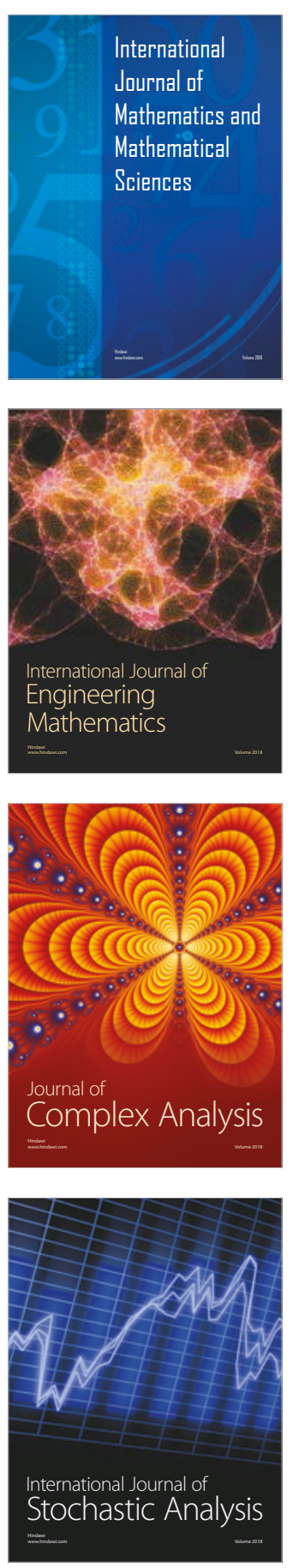
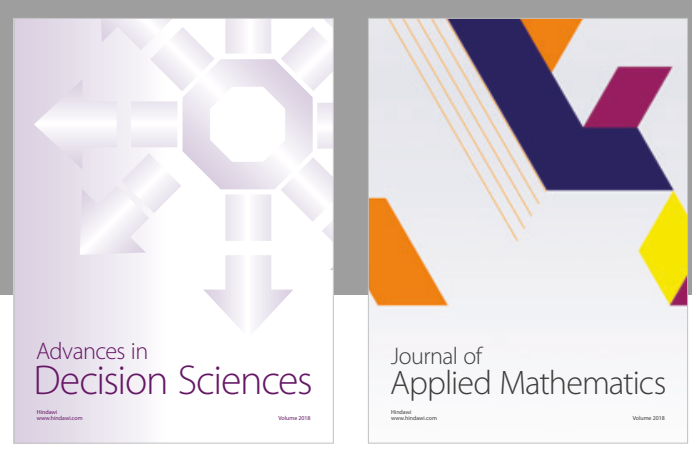

Journal of

Applied Mathematics
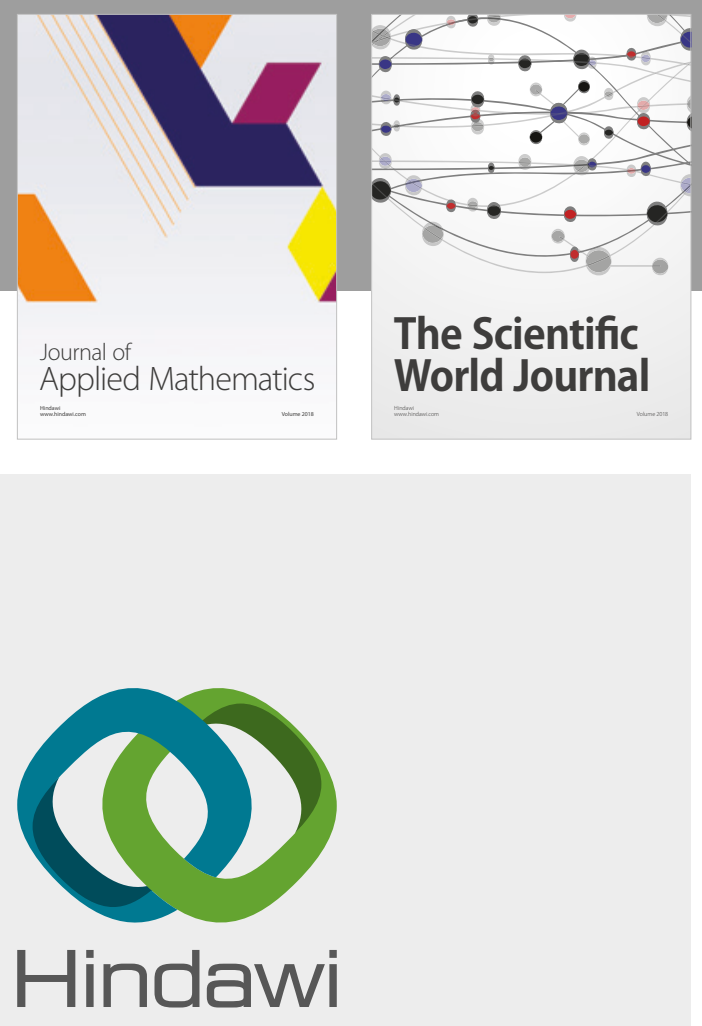

Submit your manuscripts at

www.hindawi.com

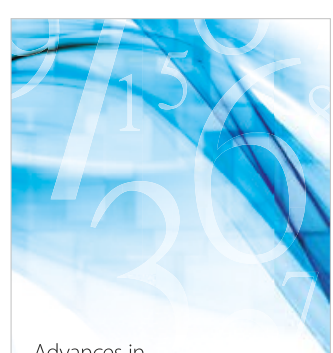

Advances in
Numerical Analysis
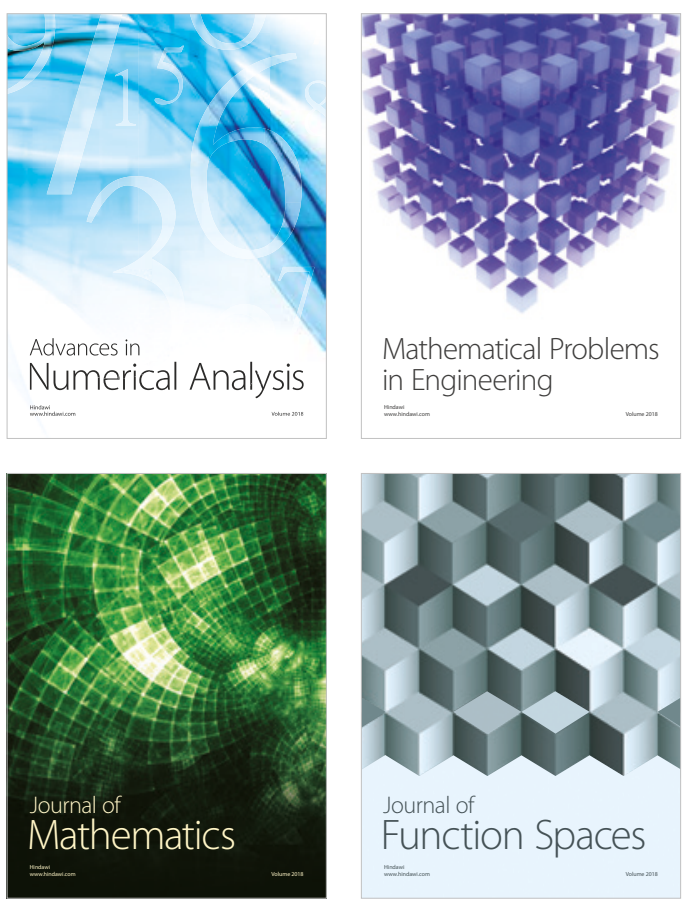

Mathematical Problems in Engineering

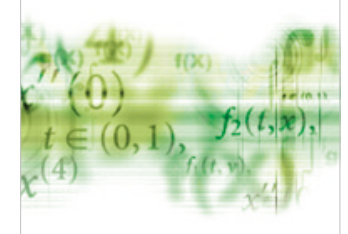

International Journal of

Differential Equations

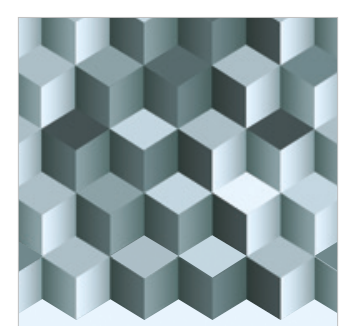

Journal of

Function Spaces

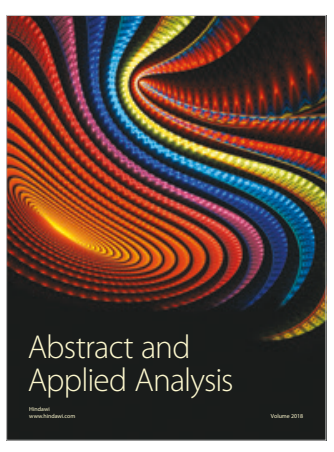

The Scientific

World Journal

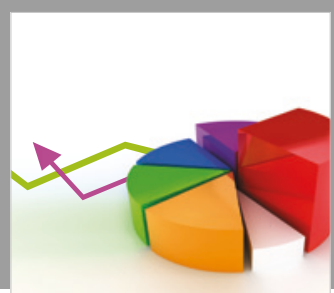

Journal of

Probability and Statistics
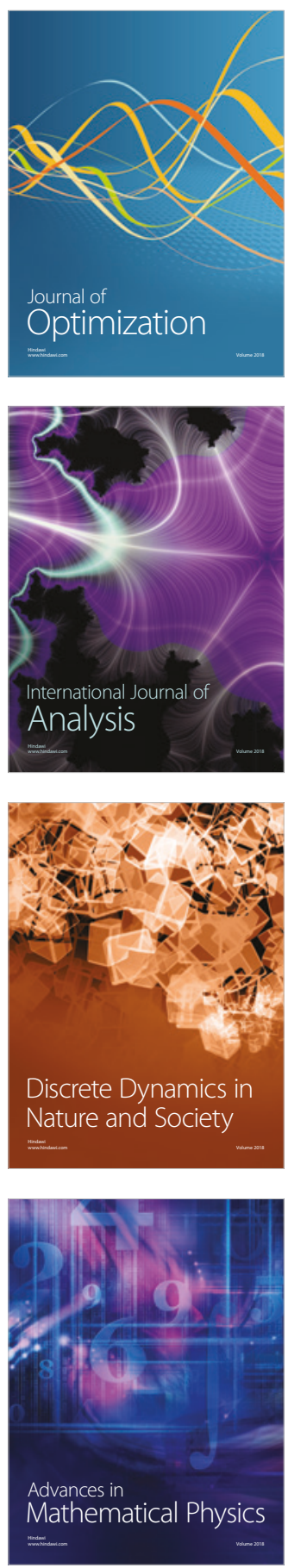